\title{
Multivariate McCormick relaxations
}

\author{
A. Tsoukalas • A. Mitsos
}

Received: 17 April 2013 / Accepted: 18 March 2014 / Published online: 2 April 2014

(C) The Author(s) 2014. This article is published with open access at Springerlink.com

\begin{abstract}
McCormick (Math Prog 10(1):147-175, 1976) provides the framework for convex/concave relaxations of factorable functions, via rules for the product of functions and compositions of the form $F \circ f$, where $F$ is a univariate function. Herein, the composition theorem is generalized to allow multivariate outer functions $F$, and theory for the propagation of subgradients is presented. The generalization interprets the McCormick relaxation approach as a decomposition method for the auxiliary variable method. In addition to extending the framework, the new result provides a tool for the proof of relaxations of specific functions. Moreover, a direct consequence is an improved relaxation for the product of two functions, at least as tight as McCormick's result, and often tighter. The result also allows the direct relaxation of multilinear products of functions. Furthermore, the composition result is applied to obtain improved convex underestimators for the minimum/maximum and the division of two functions for which current relaxations are often weak. These cases can be extended to allow composition of a variety of functions for which relaxations have been proposed.
\end{abstract}

Keywords Convex relaxation $\cdot$ Multilinear products $\cdot$ Fractional terms $\cdot$ Min/max $\cdot$ Global optimization · Subgradients

\section{Introduction}

Many nonlinear programs (NLPs), in particular in engineering design, are nonconvex and multimodal. Their global optimization typically relies on the construction of converging convex/concave relaxations, i.e., convex/concave functions that under-/over-estimate the

A. Tsoukalas · A. Mitsos

Department of Mechanical Engineering, Massachusetts Institute of Technology, Cambridge, MA, USA e-mail: tsouk@mit.edu

\section{A. Mitsos $(\varangle)$}

AVT Process Systems Engineering (SVT), RWTH Aachen University, Turmstrasse 46, 52064 Aachen, Germany

e-mail: amitsos@alum.mit.edu 
objective function and the constraints. In principle it would be desirable to construct the convex/concave envelopes, but this is typically not practical.

One approach for the convex relaxations are the so-called $\alpha \mathrm{BB}$ and $\gamma \mathrm{BB}$ relaxations, developed by Floudas and coworkers [1,2,21]. The methods are applicable to twice-continuously differential functions and rely on an estimation of the Hessian of the original functions. For elementary functions, convex/concave envelopes are known or it is possible to calculate tight relaxations. Note for instance the construction of envelopes of univariate functions described by Maranas and Floudas [22], the work by Liberti and Pantelides [20] for monomials of odd degree, and the work of Tawarmalani and Sahinidis $[46,47]$ for a class of fractional and lower semi-continuous functions.

McCormick [23,24] has provided a framework for the convex/concave relaxations of factorable functions, i.e., functions that can be represented as a finite recursive composition of binary sums, binary products and a given library of univariate intrinsic functions. The relaxations of the univariate intrinsic functions are propagated based on two main theorems, which essentially allow the relaxation of expressions in the form $F_{1} \circ f_{1}+F_{2} \circ f_{2} \cdot F_{3} \circ f_{3}$. These relaxations are in general nonsmooth [30]. If all functions involved are smooth and the convex/concave envelopes of the functions are used in the composition theorem, then the convergence order is at least quadratic [7] even if natural interval extensions with linear convergence order are used for the enclosures of functions.

An alternative to McCormick's relaxation is the auxiliary variable method (AVM) which employs auxiliary variables for each factor involved [42,48-50]. More precisely, instead of relaxing the functions, the nonconvex optimization problem is relaxed, i.e., the nonconvex problem is reformulated introducing auxiliary variables in such a way that the intrinsic functions are decoupled and can be relaxed one by one. A lower bound to the nonconvex problem is calculated via a relaxed NLP or linear program (LP).

Mitsos et al. [30] proposed the propagation of relaxations and their subgradients through procedures, thus extending the McCormick relaxations to the global optimization with algorithms embedded; examples in [30] demonstrate that optimizing in the original dimensional space can, for a class of problems, result in drastic computational savings compared to the AVM. The nonsmoothness of the relaxations implies the utilization of non-smooth optimization methods [16] for the calculation of lower bounds to the nonconvex optimization problem. The McCormick relaxations can be generalized in other ways [38], allowing also the relaxations of NLPs with dynamics (i.e., an ordinary differential equation or differential algebraic system) embedded [11,12,39,40] as well as the relaxation of implicit functions [43]. Recently, Sahlodin and Chachuat [37] also proposed the so-called McCormick-Taylor models, whereby McCormick relaxations are propagated in addition to interval bounds for enclosing the remainder term. Two implementations of McCormick's relaxations are MC++ [10] and modMC [13]; the former is freely available and used herein to calculate the McCormick relaxations.

While McCormick relaxations are clearly a very important tool, they have the limitation of only allowing univariate composition, i.e., univariate outer function. Herein, a generalization to multivariate outer functions is proposed via a reformulation of McCormick's Composition theorem in terms of a simple optimization problem. The new theorem directly allows the relaxation and subgradient propagation through procedures similar to [30] under mild assumptions. It even gives rules for the propagation of the subdifferential.

Auxiliary variable method has two clear advantages compared to McCormick's relaxations, namely that the relaxations are $(i)$ at least as tight and in some cases tighter and ( $i$ i) differentiable for a larger class of functions [48]. On the other hand, McCormick relaxations have the advantage that the relaxations are constructed in the original space and allow for 
several generalizations. The generalization of McCormick relaxations presented here, makes the relationship of the two approaches explicit, yielding a, to the best of our knowledge previously unknown, interpretation of McCormick Relaxation's as a decomposition method to solve the relaxed NLP constructed by AVM. We note that such decomposition methods for AVM have not been implemented by the global optimization community.

The proposed generalization allows a more direct relaxation of the product of functions, which proves to be at least as tight and in some cases tighter than McCormick's product rule. It also allows the direct relaxation of multilinear product of functions, i.e., without resorting to recursive application of the bilinear rule. Similarly, the proposed theorem results in at least as tight and often tighter relaxations for the minimum/maximum and the division of two functions.

The rest of the paper is organized as follows. In Sect. 2 we review McCormick's Composition Theorem and we give its generalization to multivariate outer functions, while in Sect. 3 we provide a way to propagate subgradient information. In Sect. 4 we discuss the relationship with AVM. We apply our results to compute relaxations of the product of two functions in Sect. 5, the minimum/maximum of two functions in Sect. 6 and the division of two functions in Sect. 7. We conclude and discuss future directions in Sect. 8.

\section{Convex underestimator theorems}

Theorem 1 is the main result in McCormick [23] and constructs convex/concave relaxations of composite functions where the outer function is univariate. Therein, $\operatorname{mid}(\alpha, \beta, \gamma)$ gives the median of three real numbers; in the trivial case that $\alpha=\beta=\gamma$ we have $\operatorname{mid}(\alpha, \beta, \gamma)=\alpha$; otherwise it is the numerical value that is smaller than the maximum and/or larger than the minimum.

Theorem 1 (McCormick composition theorem [23]) Let $Z \subset \mathbb{R}^{n}$ and $X \subset \mathbb{R}$ be nonempty compact convex sets. Consider the composite function $g=F \circ f(\cdot)$ where $f: Z \rightarrow \mathbb{R}$, $F: X \rightarrow \mathbb{R}$ and let $f(Z) \subset X$. Suppose that convex/concave relaxations $f^{c v}, f^{c c}: Z \rightarrow \mathbb{R}$ of $f$ on $Z$ are known. Let $F^{c v}: X \rightarrow \mathbb{R}$ be a convex relaxation of $F$ on $X$ and let $x^{\min } \in X$ be a point where $F^{c v}$ attains its minimum on $X$. Then $\bar{g}^{c v}: Z \rightarrow \mathbb{R}$,

$$
\bar{g}^{c v}(\mathbf{z})=F^{c v}\left(\operatorname{mid}\left\{f^{c v}(\mathbf{z}), f^{c c}(\mathbf{z}), x^{\min }\right\}\right),
$$

is a convex relaxation of $g$ on $Z$.

A similar theorem exists for the concave relaxation. Below we give an equivalent, yet more convenient to generalize, definition of the McCormick relaxation.

Proposition 1 Let $g^{c v}: Z \rightarrow \mathbb{R}$

$$
g^{c v}(\mathbf{z})=\min _{x \in X}\left\{F^{c v}(x) \mid f^{c v}(\mathbf{z}) \leq x \leq f^{c c}(\mathbf{z})\right\}
$$

For the function $\bar{g}^{c v}$ defined by (1) there holds

$$
\bar{g}^{c v}(\mathbf{z})=g^{c v}(\mathbf{z})
$$

for all $\mathbf{z} \in Z$.

Proof Note that we clearly have $f^{c v}(\mathbf{z}) \leq f^{c c}(\mathbf{z})$ for all $\mathbf{z} \in Z$ and that since $f(Z) \subset X$ there holds $\left[f^{c v}(\mathbf{z}), f^{c c}(\mathbf{z})\right] \cap X \neq \emptyset$ for all $\mathbf{z} \in Z$. Furthermore, let $x^{\text {min }}$ be the minimum of $F^{c v}$ in $X$. 
We consider all three cases. If

$$
\operatorname{mid}\left\{f^{c v}(\mathbf{z}), f^{c c}(\mathbf{z}), x^{\min }\right\}=x^{\min }
$$

then we have

$$
g^{c v}(z)=\min _{x \in X}\left\{F^{c v}(x) \mid f^{c v}(\mathbf{z}) \leq x \leq f^{c c}(\mathbf{z})\right\}=F^{c v}\left(x^{\min }\right)=\bar{g}^{c v}(\mathbf{z}) .
$$

If on the other hand

$$
\operatorname{mid}\left\{f^{c v}(\mathbf{z}), f^{c c}(\mathbf{z}), x^{\min }\right\}=f^{c v}(\mathbf{z})
$$

we note that $f^{c v}(\mathbf{z}) \leq f^{c c}(\mathbf{z})$ and thus $x^{\min } \leq f^{c v}(\mathbf{z}) \leq f^{c c}(\mathbf{z})$. Since $F^{c v}$ is convex it must be nondecreasing for $x \geq x^{\min }$ [30]. In addition we have $x^{\min } \leq f^{c v}(\mathbf{z}) \leq f(\mathbf{z})$ and therefore $f^{c v}(\mathbf{z}) \in X$. Thus, we have

$$
g^{c v}(\mathbf{z})=\min _{x \in X}\left\{F^{c v}(x) \mid f^{c v}(\mathbf{z}) \leq x \leq f^{c c}(\mathbf{z})\right\}=F^{c v} \circ f^{c v}(\mathbf{z})=\bar{g}^{c v}(\mathbf{z}) .
$$

Similarly, if

$$
\operatorname{mid}\left\{f^{c v}(\mathbf{z}), f^{c c}(\mathbf{z}), x^{\min }\right\}=f^{c c}(\mathbf{z})
$$

we have $f^{c v}(\mathbf{z}) \leq f^{c c}(\mathbf{z}) \leq x^{\mathrm{min}}$. Since $F^{c v}$ is convex it must be nonincreasing for $x \leq x^{\mathrm{min}}$. In addition we have $f(\mathbf{z}) \leq f^{c c}(\mathbf{z}) \leq x^{\mathrm{min}}$ and therefore $f^{c c}(\mathbf{z}) \in X$. Thus, we have

$$
g^{c v}(\mathbf{z})=\min _{x \in X}\left\{F^{c v}(x) \mid f^{c v}(\mathbf{z}) \leq x \leq f^{c c}(\mathbf{z})\right\}=F^{c v}\left(f^{c c}(\mathbf{z})\right)=\bar{g}^{c v}(\mathbf{z}) .
$$

Theorem 2 gives a generalization of Theorem 1 for multivariate outer functions. Its proof makes use of Lemma 1, which we will also use in the development of subgradient propagation in Sect. 3. Note that $\partial g(x)$ denotes the subdifferential of $g$ at $x$, i.e., the set of all subgradients.

Lemma 1 [17] Let $f_{1}, \ldots, f_{m}$ be $m$ convex functions from $\mathbb{R}^{n} \rightarrow \mathbb{R}$ and let $F$ be a convex and non-decreasing function from $\mathbb{R}^{m} \rightarrow \mathbb{R}$. Then $g(x)=F\left(f_{1}(x), \ldots, f_{m}(x)\right)$ is a convex function. Furthermore

$\partial g(x)=\left\{\sum_{i=1}^{m} \rho_{i} s_{i}:\left(\rho_{1}, \ldots, \rho_{m}\right) \in \partial F\left(f_{1}(x), \ldots, f_{m}(x)\right), s_{i} \in \partial f_{i}(x) \quad \forall i=1, \ldots, m\right\}$.

Theorem 2 Let $Z \subset \mathbb{R}^{n}$ and $X \subset \mathbb{R}^{m}$ be nonempty compact convex sets. Consider the composite function $g=F\left(f_{1}(\mathbf{z}), \ldots, f_{m}(\mathbf{z})\right)$, where $F: X \rightarrow \mathbb{R}$ and for $i \in I=\{1, \ldots, m\}$, $f_{i}: Z \rightarrow \mathbb{R}$ are continuous functions, and let

$$
\left\{\left(f_{i}(\mathbf{z}), \ldots, f_{m}(\mathbf{z})\right) \mid \mathbf{z} \in Z\right\} \subset X .
$$

Suppose that convex relaxations $f_{i}^{c v}: Z \rightarrow \mathbb{R}$ and concave relaxations $f_{i}^{c c}: Z \rightarrow \mathbb{R}$ of $f_{i}$ on $Z$ are known for every $i \in I$. Let $F^{c v}: X \rightarrow \mathbb{R}$ be a convex relaxation of $F$ on $X$ and $F^{c c}: X \rightarrow \mathbb{R}$ be a concave relaxation of $F$ on $X$. Then $g^{c v}: Z \rightarrow \mathbb{R}$,

$$
g^{c v}(\mathbf{z})=\min _{\mathbf{x} \in X}\left\{F^{c v}(\mathbf{x}) \mid f_{i}^{c v}(\mathbf{z}) \leq x_{i} \leq f_{i}^{c c}(\mathbf{z}), \quad \forall i \in I\right\}
$$

is a convex relaxation of $g$ on $Z$ and $g^{c c}: Z \rightarrow \mathbb{R}$,

$$
g^{c c}(\mathbf{z})=\max _{\mathbf{x} \in X}\left\{F^{c c}(\mathbf{x}) \mid f_{i}^{c v}(\mathbf{z}) \leq x_{i} \leq f_{i}^{c c}(\mathbf{z}), \quad \forall i \in I\right\}
$$

is a concave relaxation of $g$ on $Z$. 
Proof First we prove that $g^{c v}$ underestimates $g$ on $Z$. Using (3) and the fact that $f_{i}^{c v}(\mathbf{z}) \leq$ $f_{i}(\mathbf{z}) \leq f_{i}^{c c}(\mathbf{z})$ we obtain

$$
\begin{aligned}
g^{c v}(\mathbf{z}) & =\min _{\mathbf{x} \in X}\left\{F^{c v}(\mathbf{x}) \mid f_{i}^{c v}(\mathbf{z}) \leq x_{i} \leq f_{i}^{c c}(\mathbf{z}), \quad \forall i \in I\right\} \\
& \leq \min _{\mathbf{x} \in X}\left\{F^{c v}(\mathbf{x}) \mid x_{i}=f_{i}(\mathbf{z}), \quad \forall i \in I\right\} \\
& =F^{c v}\left(f_{1}(\mathbf{z}), \ldots, f_{m}(\mathbf{z})\right) \\
& \leq F\left(f_{1}(\mathbf{z}), \ldots, f_{m}(\mathbf{z})\right)=g(\mathbf{z}) .
\end{aligned}
$$

Next we prove that $g^{c v}$ is convex. Consider the function $h$ defined on $X \times X$ by

$$
h\left(\chi^{c v}, \chi^{c c}\right)=\min _{\mathbf{x} \in X \subset \mathbb{R}^{m}}\left\{F^{c v}(\mathbf{x}) \mid-\mathbf{x} \leq-\chi^{c v}, \mathbf{x} \leq-\chi^{c c}\right\}
$$

with $F^{c v}$ convex.

From convexity of $F^{c v}$, the function $h$ is increasing and convex as a perturbation function of a convex problem [8]. Observing that

$$
g^{c v}(\mathbf{z})=h\left(f_{1}^{c v}(\mathbf{z}), \ldots, f_{m}^{c v}(\mathbf{z}),-f_{1}^{c c}(\mathbf{z}), \ldots,-f_{m}^{c c}(\mathbf{z})\right)
$$

and applying Lemma 1 we obtain convexity of $g^{c v}$. Note, that the negative sign at the right hand side of the second constraint in the definition of $h$, was chosen conveniently to negate the concave terms $f_{i}^{c c}$ and decompose $g^{c v}$ to a convex function of convex functions. The proof for $g^{c c}$ is analogous.

We note that $g^{c v} / g^{c c}$ is not in general the convex/concave envelope of $g$ even if $F^{c v}, f^{c v}$ are the convex envelopes and $F^{c c}, f^{c c}$ the concave envelopes of $F, f$ respectively, see e.g., Fig. 1.

The definitions of $g^{c v} / g^{c c}$ at a point $\mathbf{z}$ involve the minimization/maximization of the convex/concave relaxation $F^{c v}, F^{c c}$ of $F$, where the convex/concave relaxations are computed over $X$ and the optimization is over $X \cap B$ where B is a box defined by $f_{i}^{c v}(\mathbf{z}), f_{i}^{c c}(\mathbf{z})$. This is typically a relatively easy convex problem to solve as $F^{c v}, F^{c c}$ are usually simple functions. In many cases, including the binary product of functions (Sect. 5), the solution can be described as a function of $\mathbf{z}$ in closed form.

Similarly to McCormick's relaxations, nested functions can be handled by recursive application of the theorem and do not present any difficulty. The only requirement is the availability of closed form solutions or reliable algorithms to solve the convex problems.

For the rest of the paper, unless otherwise stated, we assume that

$$
\left[f_{1}^{c v}(\mathbf{z}), f_{1}^{c c}(\mathbf{z})\right] \times \cdots \times\left[f_{m}^{c v}(\mathbf{z}), f_{m}^{c c}(\mathbf{z})\right] \subset X .
$$

Note that this is without loss of generality as we can always take

$$
\bar{f}_{i}^{c v}(\mathbf{z})=\max \left\{f_{i}^{c v}(\mathbf{z}), \min _{\mathbf{x} \in X} x_{i}\right\}, \quad \bar{f}_{i}^{c c}(\mathbf{z})=\min \left\{f_{i}^{c c}(\mathbf{z}), \max _{\mathbf{x} \in X} x_{i}\right\} .
$$

More specifically, if we assume that $X$ is a box defined as $\left[f_{1}^{L}, f_{1}^{U}\right] \times \cdots \times\left[f_{m}^{L}, f_{m}^{U}\right]$ where $\left[f_{i}^{L}, f_{i}^{U}\right]$ is an inclusion function of $f_{i}$ on $Z$ we can take

$$
\bar{f}_{i}^{c v}(\mathbf{z})=\max \left\{f_{i}^{c v}(\mathbf{z}), f_{i}^{L}\right\}, \quad \bar{f}^{c c}(\mathbf{z})=\min \left\{f_{i}^{c c}(\mathbf{z}), f_{i}^{U}\right\} .
$$

Corollary 3 gives a simplified version of Theorem 2 in the case of monotonicity, which we also utilize to compute convex/concave relaxations for the minimum/maximum of two functions, Sect. 6 . 
Corollary 3 If in addition to the assumptions of Theorem 2, Assumption (4) holds and

1. $F^{c v}$ is monotonic increasing then

$$
g^{c v}(\mathbf{z})=F^{c v}\left(f_{1}^{c v}(\mathbf{z}), . ., f_{m}^{c v}(\mathbf{z})\right)
$$

is a convex relaxation of $g$.

2. $F^{c v}$ is monotonic decreasing then

$$
g^{c v}(\mathbf{z})=F^{c v}\left(f_{1}^{c c}(\mathbf{z}), . ., f_{m}^{c c}(\mathbf{z})\right)
$$

is a convex relaxation of $g$.

3. $F^{c c}$ is monotonic increasing then

$$
g^{c c}(\mathbf{z})=F^{c c}\left(f_{1}^{c c}(\mathbf{z}), . ., f_{m}^{c c}(\mathbf{z})\right)
$$

is a concave relaxation of $g$.

4. $F^{c c}$ is monotonic decreasing then

$$
g^{c c}(\mathbf{z})=F^{c c}\left(f_{1}^{c v}(\mathbf{z}), . ., f_{m}^{c v}(\mathbf{z})\right)
$$

is a concave relaxation of $g$.

The convexity of $g^{c v}$ and concavity of $g^{c c}$ in this case is well known, e.g., [8].

\section{Subgradient propagation}

Theorem (2) allows the evaluation of the convex/concave relaxation of an arbitrary composite function at a point $\mathbf{z}$, provided that convex/concave relaxations of the intrinsic functions are available. As demonstrated in Mitsos et al. [30] the calculation of subgradients of the convex/concave relaxations is useful. In this section the results of Mitsos et al. [30] are generalized to multivariate outer-functions and to the entire subdifferential.

Lemma 2 [Adapted from strong duality theorem.] Consider the problem

$$
h\left(\chi^{c v}, \chi^{c c}\right)=\min _{\mathbf{x} \in X \subset \mathbb{R}^{m}}\left\{F^{c v}(\mathbf{x}) \mid-\mathbf{x} \leq-\chi^{c v}, \mathbf{x} \leq-\chi^{c c}\right\}
$$

with $F^{c v}$ convex. Then $\mathbf{u}=\left[\begin{array}{l}\hat{\lambda}^{c v} \\ \hat{\lambda}^{c c}\end{array}\right]$ is an optimal solution of the dual problem $D\left(\hat{\chi}^{c v}, \hat{\chi}^{c c}\right)$ given by

$$
\max _{\left(\lambda^{c v}, \lambda^{c c}\right)} \min _{\mathbf{x} \in X}\left\{F^{c v}(\mathbf{x})+\left(\lambda^{c v}\right)^{T}\left(-\mathbf{x}+\hat{\chi}^{c v}\right)+\left(\lambda^{c c}\right)^{T}\left(\mathbf{x}+\hat{\chi}^{c c}\right)\right\}
$$

at $\hat{\chi}=\left[\begin{array}{l}\hat{\chi}^{c v} \\ \hat{\chi}^{c c}\end{array}\right]$ with $\hat{\chi}^{c v} \leq-\hat{\chi}^{c c}$, if and only if $\mathbf{u} \in \partial h\left(\hat{\chi}^{c v}, \hat{\chi}^{c c}\right)$.

Proof The proof is based on the proof of the strong duality theorem in [14]. If $\mathbf{u}=\left[\begin{array}{l}\hat{\lambda}^{c v} \\ \hat{\lambda}^{c c}\end{array}\right] \geq 0$ solves the dual then

$$
\min _{\mathbf{x} \in X} F^{c v}(\mathbf{x})+\mathbf{u}^{T}\left[\begin{array}{c}
-\mathbf{x}+\hat{\chi}^{c v} \\
\mathbf{x}+\hat{\chi}^{c c}
\end{array}\right]=h\left(\hat{\chi}^{c v}, \hat{\chi}^{c c}\right) .
$$


For any fixed $\bar{\chi}=\left[\begin{array}{l}\bar{\chi}^{c v} \\ \bar{\chi}^{c c}\end{array}\right] \in \mathbb{R}^{2 m}$, if $\mathbf{x} \in X$ with $\left[\begin{array}{c}-\mathbf{x} \\ \mathbf{x}\end{array}\right] \leq\left[\begin{array}{c}-\bar{\chi}^{c v} \\ -\bar{\chi}^{c c}\end{array}\right]$, then there holds

$$
F^{c v}(\mathbf{x})-\mathbf{u}^{T}(\bar{\chi}-\hat{\chi}) \geq F^{c v}(\mathbf{x})+\mathbf{u}^{T}\left[\begin{array}{c}
-\mathbf{x}+\hat{\chi}^{c v} \\
\mathbf{x}+\hat{\chi}^{c c}
\end{array}\right] \geq h\left(\hat{\chi}^{c v}, \hat{\chi}^{c c}\right) \text {. }
$$

Thus, for fixed $\bar{\chi}$

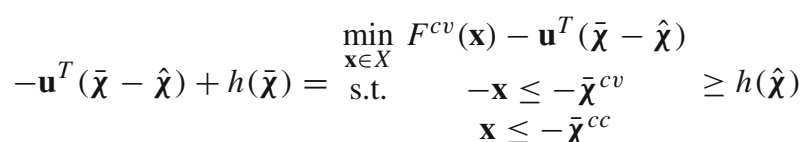

and $\mathbf{u} \in \partial h\left(\hat{\chi}^{c v}, \hat{\chi}^{c c}\right)$.

For the converse assume $\mathbf{u}=\left[\begin{array}{l}\hat{\lambda}^{c v} \\ \hat{\lambda}^{c c}\end{array}\right] \in \partial h\left(\hat{\chi}^{c v}, \hat{\chi}^{c c}\right)$. Noting that $h$ is non-decreasing, let $\mathbf{e}^{i}$ denote the $i$ th unit vector and observe

$$
h(\hat{\chi}) \geq h\left(\hat{\chi}-\mathbf{e}^{i}\right) \geq h(\hat{\chi})-u_{i}
$$

for all $i$, and thus $\mathbf{u} \geq \mathbf{0}$ and $\mathbf{u}$ is dual feasible. For any $\tilde{\mathbf{x}} \in X$ let $\tilde{\chi}=\left[\begin{array}{c}\tilde{\mathbf{x}} \\ -\tilde{\mathbf{x}}\end{array}\right]$. We have

$$
F^{c v}(\tilde{\mathbf{x}})=h(\tilde{\chi}) \geq h(\hat{\chi})+\mathbf{u}^{T}(\tilde{\chi}-\hat{\chi})=h(\hat{\chi})+\mathbf{u}^{T}\left[\begin{array}{c}
\tilde{\mathbf{x}}-\hat{\chi}^{c v} \\
-\tilde{\mathbf{x}}-\hat{\chi}^{c c}
\end{array}\right] .
$$

or rearranging

$$
h(\hat{\chi}) \leq F^{c v}(\tilde{\mathbf{x}})+\mathbf{u}^{T}\left[\begin{array}{c}
-\tilde{\mathbf{x}}+\hat{\chi}^{c v} \\
\tilde{\mathbf{x}}+\hat{\chi}^{c c}
\end{array}\right]
$$

and therefore

$$
h(\hat{\chi}) \leq \min _{\mathbf{x} \in X} F^{c v}(\mathbf{x})+\mathbf{u}^{T}\left[\begin{array}{c}
-\mathbf{x}+\hat{\chi}^{c v} \\
\mathbf{x}+\hat{\chi}^{c c}
\end{array}\right] .
$$

On the other hand, weak duality yields the opposite inequality and thus equality holds and $\mathbf{u}$ is optimal.

Theorem 4 The subdifferential of $g^{c v}$ at $\hat{\mathbf{z}}$ is given by

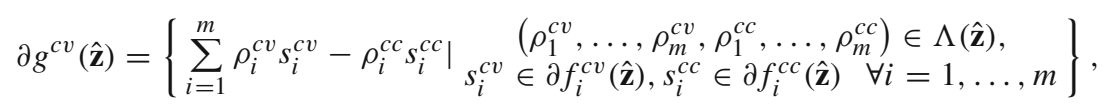

where

$$
\begin{gathered}
\Lambda(\hat{\mathbf{z}})=\underset{\left(\lambda^{c v}, \lambda^{c c}\right)}{\arg \max }\left\{\min _{\mathbf{x} \in X} L\left(\mathbf{x}, \lambda^{c v}, \lambda^{c c}, \hat{\mathbf{z}}\right)\right\} \\
L\left(\mathbf{x}, \lambda^{c v}, \lambda^{c c}, \hat{\mathbf{z}}\right)=F^{c v}(\mathbf{x})+\sum_{i=1}^{m} \lambda_{i}^{c v}\left(-\mathbf{x}+f_{i}^{c v}(\hat{\mathbf{z}})\right)+\lambda_{i}^{c c}\left(\mathbf{x}-f_{i}^{c c}(\hat{\mathbf{z}})\right)
\end{gathered}
$$

Proof The subdifferential of the convex function $-f_{i}^{c c}$ is given by

$$
\partial\left(-f_{i}^{c c}\right)(\hat{\mathbf{z}})=\left\{\mathbf{s}:-\mathbf{s} \in \partial\left(f_{i}^{c c}\right)(\hat{\mathbf{z}})\right\} .
$$

Since

$$
g^{c v}(\hat{\mathbf{z}})=h\left(f_{1}^{c v}(\hat{\mathbf{z}}), \ldots, f_{m}^{c v}(\hat{\mathbf{z}}),-f_{1}^{c c}(\hat{\mathbf{z}}), \ldots,-f_{m}^{c v}(\hat{\mathbf{z}})\right)
$$


the result follows from Lemmata 1,2.

We note that in some cases, including fractional (Sect. 7) and multilinear (Sect. 5) terms, a tighter convex relaxation $F^{c v}$ of $F$ than the ones available in closed form, can be calculated through a convex optimization problem of the form

$$
F^{c v}(\mathbf{x})=\left\{\min _{\mathbf{w} \in W} r_{1}(\mathbf{x}, \mathbf{w}) \mid \mathbf{r}_{2}(\mathbf{x}, \mathbf{w}) \leq 0\right\},
$$

with $W \subset \mathbb{R}^{n_{w}}, r_{1}: X \times W \rightarrow \mathbb{R}, \mathbf{r}_{2}: X \times W \rightarrow \mathbb{R}^{n_{r}}$. The convex underestimator of $g$ will be given by

$$
\begin{array}{cc}
g^{c v}(\mathbf{z})=\min _{\mathbf{x} \in X, \mathbf{w} \in W} & r_{1}(\mathbf{x}, \mathbf{w}) \\
\text { s.t. } & \mathbf{r}_{2}(\mathbf{x}, \mathbf{w}) \leq 0 \\
& f_{i}^{c v}(\mathbf{z}) \leq x_{i} \leq f_{i}^{c c}(\mathbf{z}), \quad \forall i
\end{array},
$$

where the defining problem has Lagrangian

$$
\bar{L}\left(\mathbf{x}, \mathbf{w}, \lambda^{c v}, \lambda^{c c}, \boldsymbol{\mu}\right)=r_{1}(\mathbf{x}, \mathbf{w})+\sum_{i \in I} \lambda_{i}^{c v}\left(f_{i}^{c v}(\mathbf{z})-x_{i}\right)+\sum_{i \in I} \lambda_{i}^{c c}\left(x_{i}-f_{i}^{c c}(\mathbf{z})\right)+\boldsymbol{\mu}^{T} \mathbf{r}_{2}(\mathbf{x}, \mathbf{w}) .
$$

We can calculate the subgradient of $g^{c v}(\mathbf{z})$ using Theorem 4 using the Lagrangian multipliers associated with the constraints $f_{i}^{c v}(\mathbf{z}) \leq x_{i} \leq f_{i}^{c c}(\mathbf{z})$. This is formalized in Proposition 2.

Proposition 2 If strong duality holds for (5) and $\left(\hat{\mathbf{x}}, \hat{\mathbf{w}},\left(\hat{\lambda}^{c v}, \hat{\lambda}^{c c}, \hat{\boldsymbol{\mu}}\right)\right)$ is an optimal primal dual pair of (5) then $\left(\hat{\mathbf{x}},\left(\hat{\lambda}^{c v}, \hat{\lambda}^{c c}\right)\right)$ is an optimal primal dual pair for the problem

$$
g^{c v}(\mathbf{z})=\min _{\mathbf{x} \in X}\left\{F^{c v}(\mathbf{x}) \mid f_{i}^{c v}(\mathbf{z}) \leq x_{i} \leq f_{i}^{c c}(\mathbf{z}), \quad \forall i \in I\right\}
$$

with Lagrangian

$$
L\left(\mathbf{x}, \lambda^{c v}, \lambda^{c c}\right)=F^{c v}(\mathbf{x})+\sum_{i \in I} \lambda_{i}^{c v}\left(f_{i}^{c v}(\mathbf{z})-x_{i}\right)+\sum_{i \in I} \lambda_{i}^{c c}\left(x_{i}-f_{i}^{c c}(\mathbf{z})\right),
$$

where the constraints defining the box $X$ are not dualized.

Proof From strong duality we have

$$
\begin{gathered}
\bar{L}\left(\hat{\mathbf{x}}, \hat{\mathbf{w}}, \hat{\lambda}^{c v}, \hat{\lambda}^{c c}, \hat{\boldsymbol{\mu}}\right)=r_{1}(\hat{\mathbf{x}}, \hat{\mathbf{w}}), \\
\mathbf{r}_{2}(\hat{\mathbf{x}}, \hat{\mathbf{w}}) \leq 0, \\
f_{i}^{c v}(\mathbf{z}) \leq \hat{x}_{i} \leq f_{i}^{c c}(\mathbf{z}) \text { for all } i, \\
\hat{\boldsymbol{\mu}}^{T} \mathbf{r}_{2}(\hat{\mathbf{x}}, \hat{\mathbf{w}})=0, \\
\hat{\lambda}_{i}^{c v}\left(f_{i}^{c v}(\mathbf{z})-\hat{x}_{i}\right)=0, \quad \hat{\lambda}_{i}^{c c}\left(\hat{x}_{i}-f_{i}^{c c}(\mathbf{z})\right)=0 \text { for all } i .
\end{gathered}
$$

Using (12), (9) we obtain

$$
L\left(\hat{\mathbf{x}}, \hat{\lambda}^{c v}, \hat{\lambda}^{c c}\right)=\bar{L}\left(\hat{\mathbf{x}}, \hat{\mathbf{w}}, \hat{\lambda}^{c v}, \hat{\lambda}^{c c}, \hat{\boldsymbol{\mu}}\right) .
$$

Keeping in mind (12) and (10) to show that $\left(\hat{\mathbf{x}},\left(\hat{\lambda}^{c v}, \hat{\lambda}^{c c}\right)\right)$ is an optimal point with its corresponding Lagrangian multipliers of (7) we only need

$$
\hat{\mathbf{x}} \in \arg \min _{\mathbf{x} \in X} L\left(\mathbf{x}, \hat{\lambda}^{c v}, \hat{\lambda}^{c c}\right) .
$$


Assume to the contrary that there exist an $\overline{\mathbf{x}} \in X$ with

$$
L\left(\overline{\mathbf{x}}, \hat{\lambda}^{c v}, \hat{\lambda}^{c c}\right)<L\left(\hat{\mathbf{x}}, \hat{\lambda}^{c v}, \hat{\lambda}^{c c}\right)
$$

and let

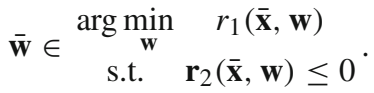

We have $r_{1}(\overline{\mathbf{x}}, \overline{\mathbf{w}})=F^{c v}(\overline{\mathbf{x}})$ and $\mathbf{r}_{2}(\overline{\mathbf{x}}, \overline{\mathbf{w}}) \leq 0$. Then we have

$$
\begin{aligned}
\bar{L}\left(\overline{\mathbf{x}}, \overline{\mathbf{w}}, \hat{\lambda}^{c v}, \hat{\lambda}^{c c}, \hat{\boldsymbol{\mu}}\right)= & r_{1}(\overline{\mathbf{x}}, \overline{\mathbf{w}})+\sum_{i \in I}\left(\hat{\lambda}_{i}^{c v}\left(f_{i}^{c v}(\mathbf{z})-\bar{x}_{i}\right)\right) \\
& +\sum_{i \in I}\left(\hat{\lambda}_{i}^{c c}\left(\bar{x}_{i}-f_{i}^{c c}(\mathbf{z})\right)\right)+\hat{\boldsymbol{\mu}}^{T} \mathbf{r}_{2}(\overline{\mathbf{x}}, \overline{\mathbf{w}}) \\
= & F^{c v}(\overline{\mathbf{x}})+\sum_{i \in I}\left(\hat{\lambda}_{i}^{c v}\left(f_{i}^{c v}(\mathbf{z})-\bar{x}_{i}\right)\right) \\
& +\sum_{i \in I}\left(\hat{\lambda}_{i}^{c c}\left(\bar{x}_{i}-f_{i}^{c c}(\mathbf{z})\right)\right)+\hat{\boldsymbol{\mu}}^{T} \mathbf{r}_{2}(\overline{\mathbf{x}}, \overline{\mathbf{w}}) \\
= & L\left(\overline{\mathbf{x}}, \hat{\lambda}^{c v}, \hat{\lambda}^{c c}\right)+\hat{\boldsymbol{\mu}}^{T} \mathbf{r}_{2}(\overline{\mathbf{x}}, \overline{\mathbf{w}}) \\
< & L\left(\hat{\mathbf{x}}, \hat{\lambda}^{c v}, \hat{\lambda}^{c c}\right)+\hat{\boldsymbol{\mu}}^{T} \mathbf{r}_{2}(\overline{\mathbf{x}}, \overline{\mathbf{w}}) \\
\leq & L\left(\hat{\mathbf{x}}, \hat{\lambda}^{c v}, \hat{\lambda}^{c c}\right) \\
= & \bar{L}\left(\hat{\mathbf{x}}, \hat{\mathbf{w}}^{c c}, \hat{\lambda}^{c v}, \hat{\lambda}^{c c}, \hat{\boldsymbol{\mu}}\right),
\end{aligned}
$$

which is a contradiction since

$$
(\hat{\mathbf{x}}, \hat{\mathbf{w}}) \in \arg \min _{\mathbf{x} \in X, \mathbf{w} \in \mathbf{W}} \bar{L}\left(\mathbf{x}, \mathbf{w}, \hat{\lambda}^{c v}, \hat{\lambda}^{c c}, \hat{\boldsymbol{\mu}}\right) .
$$

\section{McCormick relaxations and the auxiliary variable method}

In this section we revisit the relationship between McCormick relaxations and the AVM $[41,42]$. AVM lies at the heart of the state of the art software BARON [35,36], and handles composite functions implicitly by a substitution of argument functions with auxiliary variables.

While it is well known that both methods provide lower bounding mechanisms for factorable functions, the restatement of McCormick Relaxations in Theorem 1 and the subsequent generalization makes the relationship between the two approaches explicit and the occasional gap in relaxations smaller.

As mentioned in the introduction, an advantage of AVM compared to McCormick's approach is the potentially tighter bounds due to repeated terms. While multivariate McCormick can provide better bounds than univariate McCormick it can still be weaker than AVM due to the same reasons. A case where multivariate McCormick can provide tighter bounds than AVM, is if tighter convex relaxations can be made practically available through optimization problems as is the case for fractional terms discussed in Sect. 7. 
McCormick's approach allows for optimization of the bounding problem in the original space. While there is no general rule dictating that a smaller number of variables will lead to superior performance, it has been demonstrated that in a class of problems with few variables and complex expressions, operating in the original space can give a drastic improvement of CPU time [30].

To illustrate the relationship of the two methodologies, consider the functions $f_{1}: Z_{1} \subset$ $\mathbb{R}^{2} \rightarrow \mathbb{R}, f_{2}: Z_{2} \subset \mathbb{R} \rightarrow \mathbb{R}, f_{3}: Z_{3} \subset \mathbb{R} \rightarrow \mathbb{R}, f_{4}: Z_{4} \subset \mathbb{R} \rightarrow \mathbb{R}$, and the composite function

$$
g(z)=f_{1}\left(f_{3}(z), f_{4}(z)\right)+f_{2}\left(f_{3}(z)\right),
$$

$z \in Z \subset \mathbb{R}$. Assume that for all intrinsic functions $f_{i}$, convex and concave relaxations $f_{i}^{c v}$, $f_{i}^{c c}$ on $Z_{i}$ are available. Furthermore assume that $Z_{i}$ are boxes and that $Z \subset Z_{3}, Z \subset Z_{4}$, $f_{3}\left(Z_{3}\right) \times f_{4}\left(Z_{4}\right) \subset Z_{1}, f_{3}\left(Z_{3}\right) \subset Z_{2}$. Note that the univariate McCormick theorem cannot handle this directly.

To solve $\left\{\min _{z \in Z} g(z)\right\}$ AVM could formulate the problem in two different ways, depending on whether it would recognize the common term $f_{3}(z)$.

$$
\begin{aligned}
& \text { Formulation } 1 \\
& \min _{\substack{z \in Z, w_{1} \in Z_{1} \\
w_{2} \in Z_{2}, w_{3} \in Z_{3}}} \\
& \begin{array}{l}
w_{2} \in Z_{2}, w_{3} \in Z_{3} \\
w_{3}^{\prime} \in Z_{3}, w_{4} \in Z_{4}
\end{array} \\
& \text { s.t. } \\
& w_{1}=f_{1}\left(w_{3}, w_{4}\right) \\
& w_{2}=f_{2}\left(w_{3}^{\prime}\right) \\
& w_{4}=f_{4}(z) \\
& w_{3}=f_{3}(z) \\
& w_{3}^{\prime}=f_{3}(z) \\
& \min _{\substack{z \in Z, w_{1} \in Z_{1} \\
w_{2} \in Z_{2}, w_{3} \in Z_{3}}} w_{1}+w_{2} \\
& w_{4} \in Z_{4} \\
& \text { s.t. } \quad w_{1}=f_{1}\left(w_{3}, w_{4}\right) \text {. } \\
& w_{2}=f_{2}\left(w_{3}\right) \\
& w_{4}=f_{4}(z) \\
& w_{3}=f_{3}(z)
\end{aligned}
$$

with corresponding convex relaxations

$$
\begin{aligned}
& \min \\
& z \in Z, w_{1} \in Z_{1} \\
& w_{2} \in Z_{2}, w_{3} \in Z_{3} \\
& w_{3}^{\prime} \in Z_{3}, w_{4} \in Z_{4} \\
& \text { s.t. } \\
& f_{1}^{c v}\left(w_{3}, w_{4}\right) \leq w_{1} \leq f_{1}^{c c}\left(w_{3}, w_{4}^{\prime}\right) \\
& f_{2}^{c v}\left(w_{3}^{\prime}\right) \leq w_{2} \leq f_{2}^{c c}\left(w_{3}^{\prime}\right) \\
& f_{4}^{c v}(z) \leq w_{4} \leq f_{4}^{c c}(z) \\
& f_{3}^{c v}(z) \leq w_{3} \leq f_{3}^{c c}(z) \\
& f_{3}^{c v}(z) \leq w_{3}^{\prime} \leq f_{3}^{c c}(z) \\
& \text { Formulation } 2 \\
& \begin{array}{cc}
\min _{\substack{z \in Z, w_{1} \in Z_{1} \\
w_{2} \in Z_{2}, w_{3} \in Z_{3} \\
w_{4} \in Z_{4}}} & w_{1}+w_{2} \\
\text { s.t. } & f_{1}^{c v}\left(w_{3}, w_{4}\right) \\
f_{2}^{c v}\left(w_{3}\right) & \leq w_{1} \leq w_{2} \leq f_{2}^{c c}\left(w_{3}, w_{4}\right) . \\
f_{4}^{c v}(z) & \leq w_{4} \leq f_{4}^{c c}(z) \\
f_{3}^{c v}(z) & \leq w_{3} \leq f_{3}^{c c}(z)
\end{array}
\end{aligned}
$$

Formulation 2 is tighter and will likely give a better bound. It is not hard to see that multivariate McCormick will give the same bound with Formulation 1 by solving the problem

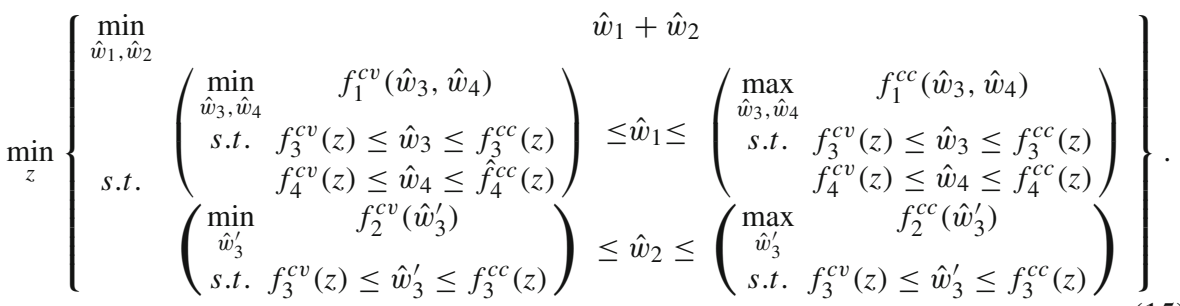

Equation (15) can be interpreted as a decomposition method of the first formulation of (14). In general all inner problems will be easy to solve analytically and a numerical algorithm 
will only be needed to minimize the resulting relaxation with respect to the original variable $z$.

In the multivariate McCormick's Framework it is possible to introduce just sufficiently mny artificial variables to improve the resulted relaxation and match the AVM. In our example this would yield the problem

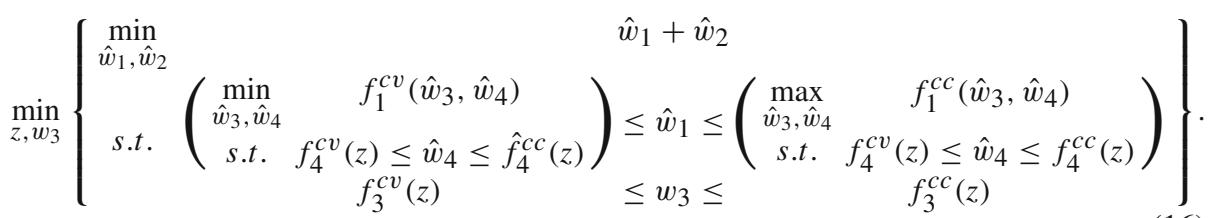

yielding the same bound with AVM while increasing the optimization space by a single variable.

It is instructive to consider only one level of composition, i.e., a function $F(f(\mathbf{z}))$, to compare the two methodologies: as it turns out, the use of a cutting plane algorithm to minimize McCormick relaxations using the subgradient propagation mechanism of Sect. 3, is strongly related to applying generalized benders decomposition [15], on the lower bounding problem defined by AVM.

To minimize $F(f(\mathbf{z}))$ AVM would formulate the problem

$$
\min _{\mathbf{x} \in X, \mathbf{z} \in Z}\left\{F^{c v}(\mathbf{x}) \mid f_{i}^{c v}(\mathbf{z}) \leq x_{i} \leq f_{i}^{c c}(\mathbf{z}), \quad \forall i \in I\right\}
$$

If we apply (generalized) Benders decomposition on (17) treating $\mathbf{z}$ as the "complicating" variables, the master problem is

$$
\min _{\mathbf{z} \in Z} V(\mathbf{z})
$$

where $V(\mathbf{z})=\max _{\lambda^{c v} \geq 0, \lambda^{c c} \geq 0} \min _{\mathbf{x} \in X}\left\{F^{c v}(\mathbf{x})+\sum_{i} \lambda_{i}^{c v}\left(f_{i}^{c v}(\mathbf{z})-\mathbf{x}\right)+\sum_{i} \lambda_{i}^{c c}\left(\mathbf{x}-f_{i}^{c c}(\mathbf{z})\right)\right\}$ for all $\mathbf{z}$.

The restricted master $V_{r}(\mathbf{z})$ employs a subset $\Lambda$ of multipliers. A $\hat{\mathbf{z}}$ obtained by solving the restricted master will be suboptimal if

$$
V_{r}(\hat{\mathbf{z}})<\max _{\lambda^{c v} \geq 0, \lambda^{c c} \geq 0} \min _{\mathbf{x} \in X}\left\{F^{c v}(\mathbf{x})+\sum_{i} \lambda_{i}^{c v}\left(f_{i}^{c v}(\hat{\mathbf{z}})-\mathbf{x}\right)+\sum_{i} \lambda_{i}^{c c}\left(\mathbf{x}-f_{i}^{c c}(\hat{\mathbf{z}})\right)\right\} .
$$

The cut obtained is

$$
V(\mathbf{z}) \geq \min _{\mathbf{x} \in X} F^{c v}(\mathbf{x})+\sum_{i} \hat{\lambda}_{i}^{c v}\left(f_{i}^{c v}(\mathbf{z})-\mathbf{x}\right)+\sum_{i} \hat{\lambda}_{i}^{c c}\left(\mathbf{x}-f_{i}^{c c}(\mathbf{z})\right),
$$

where

$$
\left(\hat{\lambda}^{c v}, \hat{\lambda}^{c c}\right) \in \underset{\lambda^{c v} \geq 0, \lambda^{c c} \geq 0}{\arg \max } \min _{\mathbf{x}}\left\{F^{c v}(\mathbf{x})+\sum_{i} \lambda_{i}^{c v}\left(f_{i}^{c v}(\hat{\mathbf{z}})-\mathbf{x}\right)+\sum_{i} \lambda_{i}^{c c}\left(\mathbf{x}-f_{i}^{c c}(\hat{\mathbf{z}})\right)\right\},
$$


cutting off $\hat{\mathbf{z}}$. The generalized-Benders cut (19) can be further relaxed by linearization around $\hat{\mathbf{z}}$, yielding,

$$
\begin{aligned}
V(\mathbf{z}) & \geq \min _{\mathbf{x} \in X} F^{c v}(\mathbf{x})+\sum_{i} \hat{\lambda}_{i}^{c v}\left(f_{i}^{c v}(\hat{\mathbf{z}})+\mathbf{s}_{i}^{c v}(\mathbf{z}-\hat{\mathbf{z}})-\mathbf{x}\right)-\sum_{i} \hat{\lambda}_{i}^{c c}\left(f_{i}^{c c}(\hat{\mathbf{z}})+\mathbf{s}_{i}^{c c}(\mathbf{z}-\hat{\mathbf{z}})-\mathbf{x}\right) \\
& =\sum_{i}\left(\hat{\lambda}_{i}^{c v} \mathbf{s}_{i}^{c v}-\hat{\lambda}_{i}^{c c} \mathbf{s}_{i}^{c c}\right)(\mathbf{z}-\hat{\mathbf{z}})+\min _{\mathbf{x} \in X} F^{c v}(\mathbf{x})+\sum_{i} \hat{\lambda}_{i}^{c v} f_{i}^{c v}(\hat{\mathbf{z}})-\sum_{i} \hat{\lambda}_{i}^{c c} f_{i}^{c c}(\hat{\mathbf{z}}) \\
& =g^{c v}(\hat{\mathbf{z}})+\sum_{i}\left(\hat{\lambda}_{i}^{c v} \mathbf{s}_{i}^{c v}-\hat{\lambda}_{i}^{c c} \mathbf{s}_{i}^{c c}\right)(\mathbf{z}-\hat{\mathbf{z}}),
\end{aligned}
$$

where $\mathbf{s}_{i}^{c v}, \mathbf{s}_{i}^{c c}$ are subgradients of $f_{i}^{c v}, f_{i}^{c c}$ at $\hat{\mathbf{z}}$. It can be seen that the linearized cut is equivalent with a subgradient inequality for $g^{c v}$ as obtained by Theorem 4. Note, that the generalized Benders' subproblem (18) generating the cut is identical with (the dual of) the problem solved to provide a function evaluation and generate the subgradient.

Therefore, in the single level composition, applying generalized Benders decomposition to AVM is equivalent to minimizing $g(\mathbf{z})$ through a first-order algorithm which at iteration $k+1$ chooses point $\mathbf{z}_{k+1}$ for evaluation by solving the linear relaxation

$$
\min _{w, \mathbf{z}}\left\{w: w \geq g\left(\mathbf{z}_{i}\right)+\mathbf{s}_{i}^{T}\left(\mathbf{z}-\mathbf{z}_{i}\right), \quad \forall i \leq k\right\},
$$

where $g\left(\mathbf{z}_{i}\right), \mathbf{s}_{i}$ are the function evaluation and subgradient returned by the oracle at iteration $i$. This is not a very efficient algorithm to minimize $g(\mathbf{z})$ and here we use it just to illustrate the equivalence. More efficient first-order methods can be found, for example, in [31].

We note, that in the (univariate and multivariate) McCormick Relaxation framework, it is straightforward to apply Theorem 4 recursively to generate subgradients for nested compositions of functions, This is not to say that it would be impossible to construct equivalent nested decomposition schemes for AVM, which has a staircase structure. In the context of stochastic programming, Birge [6] explores nested decomposition schemes for LPs. In the presence of NLPs with a staircase structure, O’Neill [32] proposes a decomposition framework combining primal and dual decomposition ideas, which is however rather involved.

Note also that the advantage of retaining the original variable space is important only in the case of such complex expressions that would need an introduction of a great number of variables to construct the AVM equivalent. Thus, the above observations on the equivalence of the two approaches for a single composition are mainly of theoretical interest.

\section{Product rule}

An interesting example of multivariate composition are products of functions. Note that bilinear terms and bilinear products of functions are very important in applications, see for instance the recent articles [19,28]. Let mult $\left(x_{1}, x_{2}\right)=x_{1} x_{2}$. As given in [3,23], the convex/concave envelopes of mult $(\cdot, \cdot)$ on $\left[x_{1}^{L}, x_{1}^{U}\right] \times\left[x_{2}^{L}, x_{2}^{U}\right]$ are

$$
\begin{aligned}
& \operatorname{mult}^{c v}=\max \left\{x_{2}^{U} x_{1}+x_{1}^{U} x_{2}-x_{1}^{U} x_{2}^{U}, x_{2}^{L} x_{1}+x_{1}^{L} x_{2}-x_{1}^{L} x_{2}^{L}\right\}, \\
& \text { mult }^{c c}=\min \left\{x_{2}^{L} x_{1}+x_{1}^{U} x_{2}-x_{1}^{U} x_{2}^{L}, x_{2}^{U} x_{1}+x_{1}^{L} x_{2}-x_{1}^{L} x_{2}^{U}\right\} .
\end{aligned}
$$

Theorem 2 directly gives convex/concave relaxations for the product of two functions. 
Corollary 5 Let $g(\mathbf{z})=\operatorname{mult}\left(f_{1}(\mathbf{z}), f_{2}(\mathbf{z})\right)$, with $f_{1}: Z \subset \mathbb{R}^{n} \rightarrow \mathbb{R}, f_{2}: Z \subset \mathbb{R}^{n} \rightarrow \mathbb{R}$. Let also $f_{i}^{L}, f_{i}^{U}$ denote bounds for $f_{i}$, i.e., $f_{i}^{L} \leq f_{i}(\mathbf{z}) \leq f_{i}^{U}$ and $f_{i}^{c v}, f_{i}^{c c}$ convex and concave relaxations of $f_{i}$ on $Z$ respectively.

$$
\begin{gathered}
g^{c v}(\mathbf{z})=\min _{x_{i} \in\left[f_{i}^{L}, f_{i}^{U}\right]} \max \left\{f_{2}^{U} x_{1}+f_{1}^{U} x_{2}-f_{1}^{U} f_{2}^{U}, f_{2}^{L} x_{1}+f_{1}^{L} x_{2}-f_{1}^{L} f_{2}^{L}\right\} \\
\text { s.t. } \\
f_{1}^{c v}(\mathbf{z}) \leq x_{1} \leq f_{1}^{c c}(\mathbf{z}) \\
f_{2}^{c v}(\mathbf{z}) \leq x_{2} \leq f_{2}^{c c}(\mathbf{z})
\end{gathered}
$$

is a convex relaxation of $g$ on $Z$ and

$$
\begin{gathered}
g^{c c}(\mathbf{z})=\max _{x_{i} \in\left[f_{i}^{L}, f_{i}^{U}\right]} \min \left\{f_{2}^{L} x_{1}+f_{1}^{U} x_{2}-f_{1}^{U} f_{2}^{L}, f_{2}^{U} x_{1}+f_{1}^{L} x_{2}-f_{1}^{L} f_{2}^{U}\right\} \\
\text { s.t. } \\
f_{1}^{c v}(\mathbf{z}) \leq x_{1} \leq f_{1}^{c c}(\mathbf{z}) \\
f_{2}^{c v}(\mathbf{z}) \leq x_{2} \leq f_{2}^{c c}(\mathbf{z})
\end{gathered}
$$

is a concave relaxation of $g$ on $Z$.

The convex relaxation for $g$ that McCormick proposed [23] is

$$
\bar{g}^{c v}(\mathbf{z})=\max \left\{\alpha_{1}(\mathbf{z})+\alpha_{2}(\mathbf{z})-f_{1}^{L} f_{2}^{L}, \beta_{1}(\mathbf{z})+\beta_{2}(\mathbf{z})-f_{1}^{U} f_{2}^{U}\right\}
$$

where

$$
\begin{aligned}
& \alpha_{1}(\mathbf{z})=\min \left\{f_{2}^{L} f_{1}^{c v}(\mathbf{z}), f_{2}^{L} f_{1}^{c c}(\mathbf{z})\right\}, \alpha_{2}(\mathbf{z})=\min \left\{f_{1}^{L} f_{2}^{c v}(\mathbf{z}), f_{1}^{L} f_{2}^{c c}(\mathbf{z})\right\}, \\
& \beta_{1}(\mathbf{z})=\min \left\{f_{2}^{U} f_{1}^{c v}(\mathbf{z}), f_{2}^{U} f_{1}^{c c}(\mathbf{z})\right\}, \beta_{2}(\mathbf{z})=\min \left\{f_{1}^{U} f_{2}^{c v}(\mathbf{z}), f_{1}^{U} f_{2}^{c c}(\mathbf{z})\right\} .
\end{aligned}
$$

The equivalent concave relaxation is

$$
\bar{g}^{c c}(\mathbf{z})=\max \left\{\gamma_{1}(\mathbf{z})+\gamma_{2}(\mathbf{z})-f_{1}^{U} f_{2}^{L}, \delta_{1}(\mathbf{z})+\delta_{2}(\mathbf{z})-f_{1}^{U} f_{2}^{U}\right\}
$$

where

$$
\begin{aligned}
& \gamma_{1}(\mathbf{z})=\max \left\{f_{2}^{L} f_{1}^{c v}(\mathbf{z}), f_{2}^{L} f_{1}^{c c}(\mathbf{z})\right\}, \gamma_{2}(\mathbf{z})=\max \left\{f_{1}^{U} f_{2}^{c v}(\mathbf{z}), f_{1}^{U} f_{2}^{c c}(\mathbf{z})\right\}, \\
& \delta_{1}(\mathbf{z})=\max \left\{f_{2}^{U} f_{1}^{c v}(\mathbf{z}), f_{2}^{U} f_{1}^{c c}(\mathbf{z})\right\}, \delta_{2}(\mathbf{z})=\max \left\{f_{1}^{L} f_{2}^{c v}(\mathbf{z}), f_{1}^{L} f_{2}^{c c}(\mathbf{z})\right\} .
\end{aligned}
$$

Proposition 3 shows that the proposed relaxations $g^{c v} / g^{c c}$ are always at least as tight as McCormick's rule $\bar{g}^{c v} / \bar{g}^{c c}$, while Fig. 1 shows that they can be tighter.

Proposition $\mathbf{3} g^{c v}(\mathbf{z}) \geq \bar{g}^{c v}(\mathbf{z})$ for all $\mathbf{z} \in Z$ and $g^{c c}(\mathbf{z}) \leq \bar{g}^{c c}(\mathbf{z})$ for all $\mathbf{z} \in Z$.

Proof Using the well-known fact, e.g., [51], that for any function $\phi(\mathbf{x}, \mathbf{y})$ defined on $\mathcal{X} \times \mathcal{Y}$ there holds

$$
\min _{\mathbf{x} \in \mathcal{X}} \max _{\mathbf{y} \in \mathcal{Y}} \phi(\mathbf{x}, \mathbf{y}) \geq \max _{\mathbf{y} \in \mathcal{Y}} \min _{\mathbf{x} \in \mathcal{X}} \phi(\mathbf{x}, \mathbf{y}),
$$

by interchanging the minimization and maximization operators we obtain

$$
\begin{aligned}
& g^{c v}(\mathbf{z}) \geq \max \left\{\begin{array}{cccc}
\min _{x_{i} \in\left[f_{i}^{L}, f_{i}^{U}\right]} & f_{2}^{U} x_{1}+f_{1}^{U} x_{2}-f_{1}^{U} f_{2}^{U} & \min _{x_{i} \in\left[f_{i}^{L}, f_{i}^{U}\right]} & f_{2}^{L} x_{1}+f_{1}^{L} x_{2}-f_{1}^{L} f_{2}^{L} \\
\text { s.t. } & f_{1}^{c v}(\mathbf{z}) \leq x_{1} \leq f_{1}^{c c}(\mathbf{z}) & \text { s.t. } & f_{1}^{c v}(\mathbf{z}) \leq x_{1} \leq f_{1}^{c c}(\mathbf{z}) \\
& f_{2}^{c v}(\mathbf{z}) \leq x_{2} \leq f_{2}^{c c}(\mathbf{z}) & & f_{2}^{c v}(\mathbf{z}) \leq x_{2} \leq f_{2}^{c c}(\mathbf{z})
\end{array}\right\}
\end{aligned}
$$

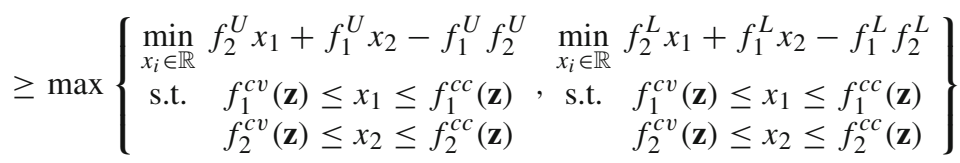

$$
\begin{aligned}
& =\max \left\{b_{1}(\mathbf{z})+b_{2}(\mathbf{z})-f_{1}^{U} f_{2}^{U}, a_{1}(\mathbf{z})+a_{2}(\mathbf{z})-f_{1}^{L} f_{2}^{L}\right\}=\bar{g}^{c v}(\mathbf{z}) \text {. }
\end{aligned}
$$




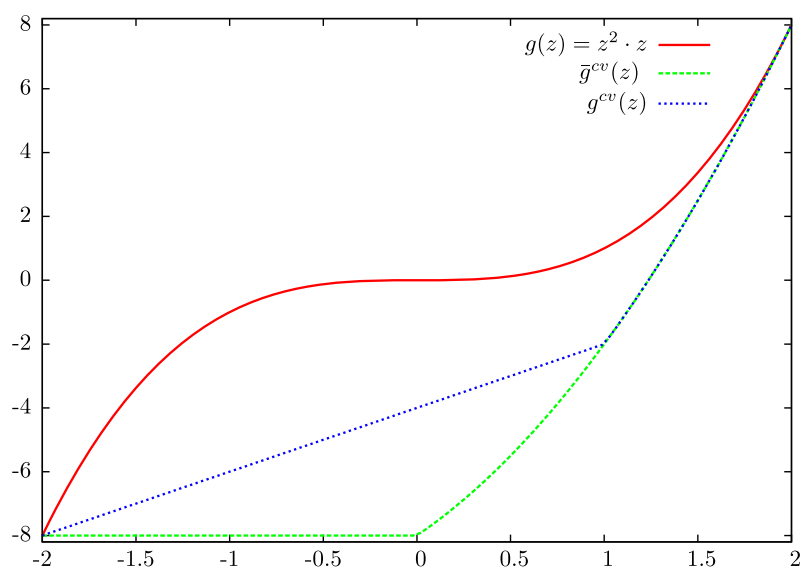

Fig. 1 Take $Z=[-2,2]$ and $g, f_{1}, f_{2}: Z \rightarrow \mathbb{R}$, such that $f_{1}(z)=z^{2}, f_{2}(z)=z, g(z)=f_{1}(z) \cdot f_{2}(z)$. The convex relaxation $g^{c v}$ of $f$ in $[-2,2]$ proposed in (20) is tighter than McCormick's relaxation $\bar{g}^{c v}$ given by Eq. (22)

The proof that $g^{c c}(\mathbf{z}) \leq \bar{g}^{c c}(\mathbf{z})$ for all $\mathbf{z} \in Z$ is similar and is omitted for brevity.

Note that the first inequality in (24) can be strict only if $f_{1}^{L}<0<f_{1}^{U}$ or $f_{2}^{L}<0<f_{2}^{U}$ and the second only if $\left[f_{1}^{c v}(\mathbf{z}), f_{1}^{c c}(\mathbf{z})\right] \not \subset\left[f_{1}^{L}, f_{1}^{U}\right]$ or $\left[f_{2}^{c v}(\mathbf{z}), f_{2}^{c c}(\mathbf{z})\right] \not \subset\left[f_{2}^{L}, f_{2}^{U}\right]$, that is, only if Assumption 4 does not hold. Scott and Barton [38] have observed that $\bar{g}^{c v}$ can be tightened by intersecting with the interval bounds. However, from the definition of $g^{c v}$ we have that $g^{c v}$ is at least as tight and in some cases tighter than the result by Scott and Barton. If $f_{1}^{U}=f_{1}^{L}$ or $f_{2}^{U}=f_{2}^{L}$, at least one of the functions is constant and the computation of the convex and concave envelopes of their product is trivial.

The convex relaxations obtained by Eqs. (20), (21) can be represented in closed form. If $f_{1}^{U}>f_{1}^{L}$ and $f_{2}^{U}>f_{2}^{L}, g^{c v}(\mathbf{z})$ can be shown to be given by

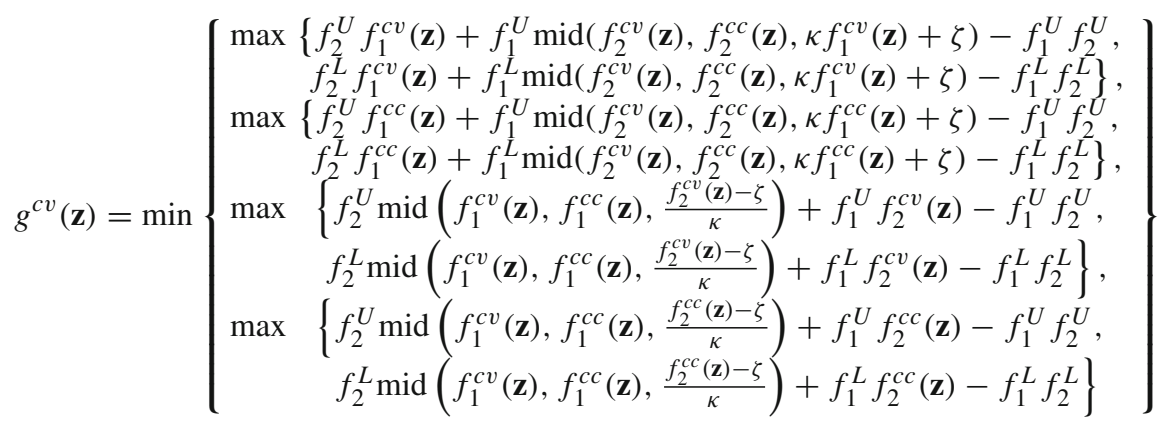

where

$$
\kappa=\frac{f_{2}^{L}-f_{2}^{U}}{f_{1}^{U}-f_{1}^{L}}, \quad \zeta=\frac{f_{1}^{U} f_{2}^{U}-f_{1}^{L} f_{2}^{L}}{f_{1}^{U}-f_{1}^{L}}
$$


Similarly, if $f_{1}^{U}>f_{1}^{L}$ and $f_{2}^{U}>f_{2}^{L}$ then $g^{c c}(\mathbf{z})$ is given by

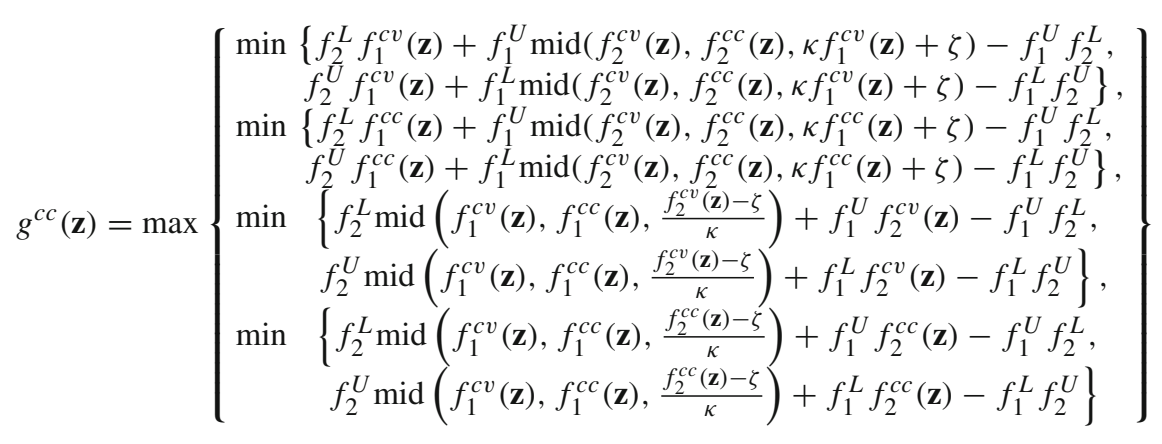

where

$$
\kappa=\frac{f_{2}^{U}-f_{2}^{L}}{f_{1}^{U}-f_{1}^{L}}, \quad \zeta=\frac{f_{1}^{U} f_{2}^{L}-f_{1}^{L} f_{2}^{U}}{f_{1}^{U}-f_{1}^{L}} .
$$

In addition to bilinear products of functions, often multilinear products of functions are used in applications. The class of functions considered herein can be summarized as $G(\mathbf{z})=$ $\sum_{t \in T} c_{t} \Pi_{i \in I_{t}} f_{i}(\mathbf{z})$, where $T$ and $I_{t} \subset I$ are index sets and $c_{t}$ are constants. Such functions can be handled by recursive application of McCormick's product rule and these approaches give weaker than possible relaxations, compare for instance [4, 5, 9, 25]. In contrast, Theorem 2 provides the framework to directly handle such terms and provide tighter relaxations. Herein, only the convex relaxations are discussed; the concave relaxations are analogous.

Rikun [34] considers $F: \mathbb{R}^{n} \rightarrow \mathbb{R}, F(\mathbf{x})=\sum_{t \in T} c_{t} \Pi_{i \in I_{t}} x_{i}$ on a hypercube $X=$ $X_{1} \times X_{2} \times \cdots \times X_{n}$, where $\left[x_{i}^{L}, x_{i}^{U}\right]$. He proves that the convex envelope $F^{c v, e n v}$ at a point $\mathbf{x}$ can be evaluated by the following optimization problem

$$
\begin{array}{r}
F^{c v, e n v}(\mathbf{x})=\min _{\lambda} \sum_{k} \lambda_{k} F\left(\mathbf{x}^{k}\right) \\
\text { s.t. } \mathbf{x}=\sum_{k} \lambda_{k} \mathbf{x}^{k} \\
\sum_{k} \lambda_{k}=1 \\
\lambda \in[0,1]^{m}
\end{array}
$$

where $\mathbf{x}^{k}$ denote the vertices of $X$. Note that this is a LP, albeit of size $m$. Note also that explicit representations exist for subclasses of this function such as the explicit facets of trilinear terms by Meyer and Floudas [25].

By Theorem 2 a convex relaxation of $G$ on $Z$ can be constructed as

$$
\begin{array}{ll}
g^{c v}(\mathbf{z})=\min _{\mathbf{x}} \quad F^{c v, e n v}(\mathbf{x}) \\
& f_{i}^{c v}(\mathbf{z}) \leq x_{i} \leq f_{i}^{c c}(\mathbf{z}), \quad i \in I .
\end{array}
$$


Noting that $\min _{y} \min _{w} h(y, w)=\min _{y, w} h(y, w)$ we obtain

$$
\begin{gathered}
g^{c v}(\mathbf{z})=\min _{\mathbf{x}, \lambda} \quad \sum_{k} \lambda_{k} F\left(\mathbf{x}^{\mathbf{k}}\right) \\
\mathbf{x}=\sum_{k} \lambda_{k} \mathbf{x}^{k} \\
\sum_{k} \lambda_{k}=1 \\
f_{i}^{c v}(\mathbf{z}) \leq x_{i} \leq f_{i}^{c c}(\mathbf{z}), \quad i \in I .
\end{gathered}
$$

which is still an LP of similar size.

By Proposition 2, Theorem 4 can still be used for the computation of subgradients if we take into account in the construction of $\sigma^{c v}$ only the Lagrangian multipliers associated with the constraints $f_{i}^{c v}(\mathbf{z}) \leq x_{i} \leq f_{i}^{c c}(\mathbf{z})$.

\section{Convex/concave envelopes and relaxations of $\mathrm{min} / \mathrm{max}$ operators}

The operators min and max often arise in engineering optimization formulations. It is wellknown that the minimum of concave functions is a concave function, but the same does not hold in general for convex functions. To the authors' best knowledge relaxations for such functions are not available in literature or in most numerical codes. For instance, the operators min / max are currently not handled by the state-of-the-art general-purpose solvers BARON [36,49] and ANTIGONE [27-29], while in MC++ [10] they are handled using the well-known reformulation

$$
\min \left(f_{1}(\mathbf{z}), f_{2}(\mathbf{z})\right)=\frac{1}{2}\left(f_{1}(\mathbf{z})+f_{2}(\mathbf{z})-\left|f_{1}(\mathbf{z})-f_{2}(\mathbf{z})\right|\right)
$$

and applying the univariate McCormick composition theorem to the negative absolute value. However, the constructed relaxations are not as tight as the ones proposed here.

Calculating interval enclosures for the function $\min \left(f_{1}(x), f_{2}(x)\right)$ given interval enclosures for $f_{1}$ and $f_{2}$ is straightforward and is also done in MC++ [10].

Proposition 4 Consider $Z \in \mathbb{R}^{n}$ and $f_{1}, f_{2}: Z \rightarrow \mathbb{R}$. Suppose that interval enclosures are given for $f_{1}$ and $f_{2}$ on $Z$, i.e., bounds $f_{1}^{L}, f_{1}^{U}, f_{2}^{L}, f_{2}^{U}$ such that

$$
f_{1}^{L} \leq f_{1}(\mathbf{z}) \leq f_{1}^{U} \quad f_{2}^{L} \leq f_{2}(\mathbf{z}) \leq f_{2}^{U}
$$

Then we have

$$
\min \left(f_{1}^{L}, f_{2}^{L}\right) \leq \min \left(f_{1}(\mathbf{z}), f_{2}(\mathbf{z})\right) \leq \min \left(f_{1}^{U}, f_{2}^{U}\right)
$$

It is noteworthy that these bounds are not exact, as shown in the next example

Example 1 Take $\min (z,-z)$ with $z \in[-1,1]$. The range is clearly $[-1,0]$ and the rule given in Proposition 4 gives a valid but overestimated enclosure as $[-1,1]$.

We can utilize Corollary 3 to compute convex/concave relaxations for the minimum/maximum of two functions. The computation of convex/concave relaxations of the minimum/maximum of two functions by Theorem 2 requires the convex/concave envelopes of $\min \left(x_{1}, x_{2}\right) / \max \left(x_{1}, x_{2}\right)$ on an arbitrary rectangle, which is easy to derive to the authors' best knowledge is not explicitly available in the literature. 
Lemma 3 Consider $Z=X_{1} \times X_{2} \subset \mathbb{R}^{2}$ with $X_{1}=\left[x_{1}^{L}, x_{1}^{U}\right]$ and $X_{2}=\left[x_{2}^{L}, x_{2}^{U}\right]$ and let $\mathbf{z}=\left(x_{1}, x_{2}\right)$. The convex envelope of $\min \left(x_{1}, x_{2}\right)$ on $Z$ is given by $\min ^{c v}: Z \rightarrow \mathbb{R}$,

$$
\begin{aligned}
\min ^{\mathrm{cv}}\left(x_{1}, \mathrm{x}_{2}\right)= & \max \left(\min ^{\mathrm{cv}, 1}\left(\mathrm{x}_{1}, \mathrm{x}_{2}\right), \min ^{\mathrm{cv}, 2}\left(\mathrm{x}_{1}, \mathrm{x}_{2}\right)\right) \text { with } \\
\min ^{\mathrm{cv}, 1}\left(\mathrm{x}_{1}, \mathrm{x}_{2}\right)= & \min \left(x_{1}^{L}, x_{2}^{L}\right)+\frac{x_{1}-x_{1}^{L}}{x_{1}^{U}-x_{1}^{L}}\left(\min \left(x_{1}^{U}, x_{2}^{L}\right)-\min \left(x_{1}^{L}, x_{2}^{L}\right)\right) \\
& +\frac{x_{2}-x_{2}^{L}}{x_{2}^{U}-x_{2}^{L}}\left(\min \left(x_{1}^{L}, x_{2}^{U}\right)-\min \left(x_{1}^{L}, x_{2}^{L}\right)\right) \\
\min ^{\mathrm{cv}, 2}\left(\mathrm{x}_{1}, \mathrm{x}_{2}\right)= & \min \left(x_{1}^{U}, x_{2}^{U}\right)+\frac{x_{1}-x_{1}^{U}}{x_{1}^{L}-x_{1}^{U}}\left(\min \left(x_{1}^{L}, x_{2}^{U}\right)-\min \left(x_{1}^{U}, x_{2}^{U}\right)\right) \\
& +\frac{x_{2}-x_{2}^{U}}{x_{2}^{L}-x_{2}^{U}}\left(\min \left(x_{1}^{U}, x_{2}^{L}\right)-\min \left(x_{1}^{U}, x_{2}^{U}\right)\right)
\end{aligned}
$$

and the concave envelope of $\max \left(x_{1}, x_{2}\right)$ on $Z$ is given by $\max ^{c c}: Z \rightarrow \mathbb{R}$,

$$
\begin{aligned}
\max ^{\mathrm{cc}}\left(\mathrm{x}_{1}, \mathrm{x}_{2}\right)= & \min \left(\max ^{\mathrm{cc}, 1}\left(\mathrm{x}, \mathrm{x}_{2}\right), \max ^{\mathrm{cc}, 2}\left(\mathrm{x}_{1}, \mathrm{x}_{2}\right)\right) \text { with } \\
\max ^{\mathrm{cc}, 1}\left(\mathrm{x}_{1}, \mathrm{x}_{2}\right)= & \max \left(x_{1}^{L}, x_{2}^{L}\right)+\frac{x_{1}-x_{1}^{L}}{x_{1}^{U}-x_{1}^{L}}\left(\max \left(x_{1}^{U}, x_{2}^{L}\right)-\max \left(x_{1}^{L}, x_{2}^{L}\right)\right) \\
& +\frac{x_{2}-x_{2}^{L}}{x_{2}^{U}-x_{2}^{L}}\left(\max \left(x_{1}^{L}, x_{2}^{U}\right)-\max \left(x_{1}^{L}, x_{2}^{L}\right)\right) \\
\max ^{\mathrm{cc}, 2}\left(\mathrm{x}_{1}, \mathrm{x}_{2}\right)= & \max \left(x_{1}^{U}, x_{2}^{U}\right)+\frac{x_{1}-x_{1}^{U}}{x_{1}^{L}-x_{1}^{U}}\left(\max \left(x_{1}^{L}, x_{2}^{U}\right)-\max \left(x_{1}^{U}, x_{2}^{U}\right)\right) \\
& +\frac{x_{2}-x_{2}^{U}}{x_{2}^{L}-x_{2}^{U}}\left(\max \left(x_{1}^{U}, x_{2}^{L}\right)-\max \left(x_{1}^{U}, x_{2}^{U}\right)\right)
\end{aligned}
$$

Proof The proof is in the Appendix.

A convex relaxation of the maximum of two functions is trivially given by the maximum of the convex relaxations of the two functions and a concave relaxation of the minimum of two functions as the minimum of the concave relaxations of the two functions.

Proposition 5 Consider $Z \in \mathbb{R}^{n}$ and $g_{1}, g_{2}, f_{1}, f_{2}: Z \rightarrow \mathbb{R}$ such that $g_{1}(\mathbf{z})=$ $\min \left(f_{1}(\mathbf{z}), f_{2}(\mathbf{z})\right), g_{2}(\mathbf{z})=\max \left(f_{1}(\mathbf{z}), f_{2}(\mathbf{z})\right)$. Suppose that interval enclosures are given for $f_{1}$ and $f_{2}$ on $Z$, i.e., bounds $f_{1}^{L}, f_{1}^{U}, f_{2}^{L}, f_{2}^{U}$ such that

$$
f_{1}^{L} \leq f_{1}(\mathbf{z}) \leq f_{1}^{U} \quad f_{2}^{L} \leq f_{2}(\mathbf{z}) \leq f_{2}^{U}
$$

and convex and concave relaxations such that

$$
f_{1}^{c v}(\mathbf{z}) \leq f_{1}(\mathbf{z}) \leq f_{1}^{c c}(\mathbf{z}) \quad f_{2}^{c v}(\mathbf{z}) \leq f_{2}(\mathbf{z}) \leq f_{2}^{c c}(\mathbf{z}) .
$$

Recall that Proposition 4 gives interval enclosures for $f$ on $Z$. The following procedure defines a convex relaxation $g_{1}^{c v}: Z \rightarrow \mathbb{R}$ of $g_{1}$ on $Z$.

If $f_{1}^{U} \leq f_{2}^{L}$ then $g_{1}^{c v}(\mathbf{z})=f_{1}^{c v}(\mathbf{z})$. Similarly, if $f_{2}^{U} \leq f_{1}^{L}$ then $g_{1}^{c v}(\mathbf{z})=f_{2}^{c v}(\mathbf{z})$. Otherwise

$$
g_{1}^{c v}(\mathbf{z})=\max \left(g_{1}^{c v, 1}(\mathbf{z}), g_{1}^{c v, 2}(\mathbf{z})\right)
$$


where

$$
\begin{aligned}
g_{1}^{c v, 1}(\mathbf{z})= & \min \left(f_{1}^{L}, f_{2}^{L}\right)+\frac{f_{1}^{c v}(\mathbf{z})-f_{1}^{L}}{f_{1}^{U}-f_{1}^{L}}\left(\min \left(f_{1}^{U}, f_{2}^{L}\right)-\min \left(f_{1}^{L}, f_{2}^{L}\right)\right) \\
& +\frac{f_{2}^{c v}(\mathbf{z})-f_{2}^{L}}{f_{2}^{U}-f_{2}^{L}}\left(\min \left(f_{1}^{L}, f_{2}^{U}\right)-\min \left(f_{1}^{L}, f_{2}^{L}\right)\right) \\
g_{1}^{c v, 2}(\mathbf{z})= & \min \left(f_{1}^{U}, f_{2}^{U}\right)+\frac{f_{1}^{c v}(\mathbf{z})-f_{1}^{U}}{f_{1}^{L}-f_{1}^{U}}\left(\min \left(f_{1}^{L}, f_{2}^{U}\right)-\min \left(f_{1}^{U}, f_{2}^{U}\right)\right) \\
& +\frac{f_{2}^{c v}(\mathbf{z})-f_{2}^{U}}{f_{2}^{L}-f_{2}^{U}}\left(\min \left(f_{1}^{U}, f_{2}^{L}\right)-\min \left(f_{1}^{U}, f_{2}^{U}\right)\right) .
\end{aligned}
$$

Furthermore, the following procedure defines a concave relaxation $g_{2}^{c c}: Z \rightarrow \mathbb{R}$ of $g_{2}$ on Z. If $f_{1}^{U} \leq f_{2}^{L}$ then $g_{2}^{c c}(\mathbf{z})=f_{1}^{c c}(\mathbf{z})$. Similarly, if $f_{2}^{U} \leq f_{1}^{L}$ then $g_{2}^{c c}(\mathbf{z})=f_{2}^{c c}(\mathbf{z})$. Otherwise $g_{2}^{c c}(\mathbf{z})=\min \left(g_{2}^{c c, 1}(\mathbf{z}), g_{2}^{c c, 2}(\mathbf{z})\right)$ where

$$
\begin{aligned}
g_{2}^{c c, 1}(\mathbf{z})= & \max \left(f_{1}^{L}, f_{2}^{L}\right)+\frac{f_{1}^{c c}(\mathbf{z})-f_{1}^{L}}{f_{1}^{U}-f_{1}^{L}}\left(\max \left(f_{1}^{U}, f_{2}^{L}\right)-\max \left(f_{1}^{L}, f_{2}^{L}\right)\right) \\
& +\frac{f_{2}^{c c}(\mathbf{z})-f_{2}^{L}}{f_{2}^{U}-f_{2}^{L}}\left(\max \left(f_{1}^{L}, f_{2}^{U}\right)-\max \left(f_{1}^{L}, f_{2}^{L}\right)\right) \\
g_{2}^{c c, 2}(\mathbf{z})= & \max \left(f_{1}^{U}, f_{2}^{U}\right)+\frac{f_{1}^{c c}(\mathbf{z})-f_{1}^{U}}{f_{1}^{L}-f_{1}^{U}}\left(\max \left(f_{1}^{L}, f_{2}^{U}\right)-\max \left(f_{1}^{U}, f_{2}^{U}\right)\right) \\
& +\frac{f_{2}^{c c}(\mathbf{z})-f_{2}^{U}}{f_{2}^{L}-f_{2}^{U}}\left(\max \left(f_{1}^{U}, f_{2}^{L}\right)-\max \left(f_{1}^{U}, f_{2}^{U}\right)\right)
\end{aligned}
$$

Proof Since $\min (\cdot, \cdot)$ and $\max (\cdot, \cdot)$ are monotonic increasing the result follows by Corollary 3.

Note that there is no guarantee that the proposed relaxation is the envelope even if the estimators of the factors are, as shown in Fig. 2.

Reformulating $\min (\cdot, \cdot)$ and $\max (\cdot, \cdot)$ operators using the absolute value of the difference, results in weak natural interval extensions and also weaker McCormick relaxations as shown in Proposition 6. Figure 2 shows that the inequality in Proposition 6 can be strict.

Proposition 6 Consider $Z \in \mathbb{R}^{n}$ and $f_{1}, f_{2}: Z \rightarrow \mathbb{R}$ such that $g_{1}(\mathbf{z})=\min \left(f_{1}(\mathbf{z}), f_{2}(\mathbf{z})\right)$. Suppose that interval enclosures are given for $f_{1}$ and $f_{2}$ on $Z$, i.e., bounds $f_{1}^{L}, f_{1}^{U}, f_{2}^{L}, f_{2}^{U}$ such that

$$
f_{1}^{L} \leq f_{1}(\mathbf{z}) \leq f_{1}^{U} \quad f_{2}^{L} \leq f_{2}(\mathbf{z}) \leq f_{2}^{U}
$$

and convex/concave relaxations such that

$$
f_{1}^{c v}(\mathbf{z}) \leq f_{1}(\mathbf{z}) \leq f_{1}^{c c}(\mathbf{z}) \quad f_{2}^{c v}(\mathbf{z}) \leq f_{2}(\mathbf{z}) \leq f_{2}^{c c}(\mathbf{z}) .
$$

For the overlapping case $f_{1}^{L}<f_{2}^{U}, f_{2}^{L}<f_{1}^{U}$, the convex/concave relaxations for min/max proposed in Theorem 5 are at least as tight as the ones obtained by McCormick's composition Theorem applied to the reformulation via the absolute value.

Proof The proof is given in the Appendix 


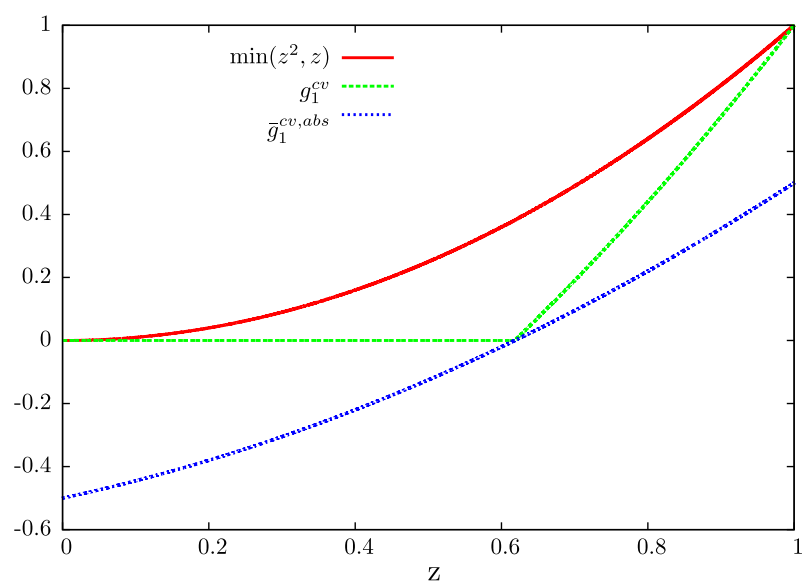

Fig. 2 Take $Z=[0,1]$ and $g_{1}, f_{1}, f_{2}: Z \rightarrow \mathbb{R}$, such that $f_{1}(z)=z^{2}, f_{2}(z)=z, g_{1}(z)=\min \left(f_{1}(z), f_{2}(z)\right)$. Note that both $f_{1}$ and $f_{2}$ have range $[0,1]$ and that both are convex and as such $f_{1}^{c v}=f_{1}$ and $f_{2}^{c v}=f_{2}$ are the convex envelopes. We have $g_{1}(z)=z^{2}$ and this is its convex envelope. It follows from Proposition 5 that $g_{1}^{c v}: Z \rightarrow \mathbb{R}, g_{1}^{c v}(z)=\max \left(0, z^{2}+z-1\right)$ is a convex relaxation of $g_{1}$ on $Z$. Note that it does not give the envelope although envelopes are used for the factors. The reformulation via the absolute value furnish a valid but less tight relaxation $\bar{g}_{1}^{c v, a b s}$

Relaxations of $\min \left(f_{1}, \ldots, f_{m}\right)$ can be computed either recursively, or by direct application of Theorem 2 if an envelope/relaxation of $\min \left(x_{1}, \ldots, x_{m}\right)$ is available on the appropriate domain. In $[0,1]^{m}$, for example, it can be shown that the convex envelope of $\min \left(x_{1}, \ldots, x_{m}\right)$ is $\max \left(0, \sum_{i} x_{i}-n+1\right)$.

If the relaxation for the multiterm operator is the envelope, direct application of the multivariate composition will result in at least as tight relaxations. If in contrast the relaxations for the multiterm operator are weak, it may be advisable to use the bivariate composition recursively.

\section{Fractional terms}

Fractional terms $f_{1}(\mathbf{z}) / f_{2}(\mathbf{z})$ often arise in engineering optimization formulations. In McCormick relaxation framework, e.g., in MC++ [10] they are handled rigorously using the presentation as $f_{1}(\mathbf{z}) \times\left(f_{2}(\mathbf{z})\right)^{-1}$, i.e., as a bilinear product with the inverse function embedded. The multivariate composition theorem can handle the fractional terms more naturally and yields at least as tight and often tighter relaxations. For the rest of this section we assume that $f_{2}^{L}>0$ or $f_{2}^{U}<0$, so that the division is well defined.

Consider the fractional term $\frac{x_{1}}{x_{2}}$ on $X_{1} \times X_{2}=\left[x_{1}^{L}, x_{1}^{U}\right] \times\left[x_{2}^{L}, x_{2}^{U}\right]$ which we will denote via the division function $\operatorname{div}(\cdot, \cdot)$. Tawarmalani and Sahinidis [47] discuss convex relaxations and the envelope for the positive orthant, i.e., for $x_{1}^{L}>0, x_{2}^{L}>0$. One relaxation by Zamora and Grossmann $[53,54]$ is given by

$$
\operatorname{div}^{c v, Z \& G}\left(x_{1}, x_{2}\right)=\frac{1}{x_{2}}\left(\frac{x_{1}+\sqrt{x_{1}^{L} x_{1}^{U}}}{\sqrt{x_{1}^{L}}+\sqrt{x_{1}^{U}}}\right)^{2} .
$$


The function $\operatorname{div}^{c v, Z \& G}$ is the convex envelope when $x_{2}^{L} \rightarrow 0$ and $x_{2}^{U} \rightarrow \infty$. A piecewise linear relaxation of div $[33,47]$ is given by

$$
\operatorname{div}^{c v, \operatorname{lin}}\left(x_{1}, x_{2}\right)=\max \left\{\frac{x_{1} x_{2}^{U}-x_{1}^{L} x_{2}+x_{1}^{L} x_{2}^{U}}{\left(x_{2}^{U}\right)^{2}}, \frac{x_{1} x_{2}^{L}-x_{1}^{U} x_{2}+x_{1}^{U} x_{2}^{L}}{\left(x_{2}^{L}\right)^{2}}\right\} .
$$

Another method to obtain a valid convex relaxation of $\operatorname{div}(\cdot, \cdot)$ on $X_{1} \times X_{2}$, assuming that either $x_{2}^{U}<0$ or $x_{2}^{L}>0$ is to apply the product rule of McCormick [23] (defined in Eq. (22)) using the representation $x_{1} \times \operatorname{Inv}\left(x_{2}\right)$ where $\operatorname{Inv}(\cdot)=(\cdot)^{-1}$. Let $\operatorname{Inv}^{L}, \operatorname{Inv}^{U}$ denote the implied bounds, and $\operatorname{Inv}^{c v}\left(x_{2}\right), \operatorname{Inv}^{c c}\left(x_{2}\right)$ the convex and concave relaxations of Inv on $X_{2}$. It is easy to verify that the result is

$$
\operatorname{div}^{c v, m c}\left(x_{1}, x_{2}\right)=\max \left\{\begin{array}{c}
\operatorname{Inv}^{L} x_{1}+\min \left\{x_{1}^{L} \operatorname{Inv}^{c v}\left(x_{2}\right), x_{1}^{L} \operatorname{Inv}^{c c}\left(x_{2}\right)\right\}-x_{1}^{L} \operatorname{Inv}^{L}, \\
\operatorname{Inv}^{U} x_{1}+\min \left\{x_{1}^{U} \operatorname{Inv}^{c v}\left(x_{2}\right), x_{1}^{U} \operatorname{Inv}^{c c}\left(x_{2}\right)\right\}-x_{1}^{U} \operatorname{Inv}^{U}
\end{array}\right\}
$$

which for the positive orthant reduces to

$$
\operatorname{div}^{c v, m c,+}\left(x_{1}, x_{2}\right)=\max \left\{\frac{x_{1}}{x_{2}^{U}}+\frac{x_{1}^{L}}{x_{2}}-\frac{x_{1}^{L}}{x_{2}^{U}}, \frac{x_{1}}{x_{2}^{L}}+\frac{x_{1}^{U}}{x_{2}}-\frac{x_{1}^{U}}{x_{2}^{L}}\right\},
$$

as computed by Quesada and Grossman [33] following the same procedure. It is shown in [33] that $\operatorname{div}^{c v, l i n}$ is a linearization of $\operatorname{div}^{c v, m c,+}$ at $x_{2}^{L}, x_{2}^{U}$ and thus

$$
\operatorname{div}^{c v, l i n}\left(x_{1}, x_{2}\right) \leq \operatorname{div}^{c v, m c,+}\left(x_{1}, x_{2}\right) \text {. }
$$

The concave envelope for the positive orthant is computed in [46] to be

$$
\operatorname{div}^{c c, m c,+}{ }_{\left(x_{1}, x_{2}\right)}=\frac{1}{x_{2}^{L} x_{2}^{U}} \min \left\{x_{2}^{U} x_{1}-x_{1}^{L} x_{2}+x_{1}^{L} x_{2}^{L}, x_{2}^{L} x_{1}-x_{1}^{U} x_{2}+x_{1}^{U} x_{2}^{U}\right\} .
$$

Finally, Tawarmalani and Sahinidis $[46,47]$ prove that the convex envelope at a point can be evaluated by solving an optimization problem

$$
\begin{aligned}
\operatorname{div}^{c v, e n v}\left(x_{1}, x_{2}\right)=\min _{y_{p}, z_{p}, z_{c}^{e}, \lambda} & z_{c}^{e} \\
\text { s.t. } \quad & z_{p} y_{p} \geq x_{1}^{L}(1-\lambda)^{2} \\
& \left(z_{c}^{e}-z_{p}\right)\left(x_{2}-y_{p}\right)=x_{1}^{U} \lambda^{2} \\
& y_{p} \geq x_{2}^{L}(1-\lambda) \\
& y_{p} \geq x_{2}-x_{2}^{U} \lambda \\
& y_{p} \leq x_{2}^{U}(1-\lambda) \\
& y_{p} \leq x_{2}-x_{2}^{L} \lambda \\
& x_{1}=x_{1}^{L}+\left(x_{1}^{U}-x_{1}^{L}\right) \lambda \\
& z_{c}^{e} \geq z_{p} \\
& \lambda \in[0,1], z_{p} \geq 0
\end{aligned}
$$

which can be reformulated as a semi-definite program. 
In $\mathrm{MC}++[10]$ a relaxation for $G(\mathbf{z})=\frac{f_{1}(\mathbf{z})}{\mathrm{f}_{2}(\mathbf{z})}$ is obtained using the representation $f_{1}(\mathbf{z}) \times$ $\left(\right.$ Inv $\left.\circ f_{2}(\mathbf{z})\right)$ and applying first McCormick's composition theorem to obtain $\left(\operatorname{Inv} \circ \mathrm{f}_{2}\right)^{\mathrm{cv}}(\mathbf{z})$, $\left(\operatorname{Inv} \circ \mathrm{f}_{2}\right)^{c c}(\mathbf{z})$ and then McCormick's product rule (22). The resulting relaxation is given by

$$
\begin{aligned}
\bar{g}^{c v, M C++}(\mathbf{z}) \\
=\max \left\{\begin{array}{l}
\min \left\{\frac{1}{f_{2}^{U}} f_{1}^{c v}(\mathbf{z}), \frac{1}{f_{2}^{U}} f_{1}^{c c}(\mathbf{z})\right\}+\min \left\{f_{1}^{L}\left(\operatorname{Inv} \circ f_{2}\right)^{c v}(\mathbf{z}), f_{1}^{L}\left(\operatorname{Inv} \circ f_{2}\right)^{c c}(\mathbf{z})\right\}-\frac{f_{1}^{L}}{f_{2}^{U}}, \\
\min \left\{\frac{1}{f_{2}^{L}} f_{1}^{c v}(\mathbf{z}), \frac{1}{f_{2}^{L}} f_{1}^{c c}(\mathbf{z})\right\}+\min \left\{f_{1}^{U}\left(\operatorname{Inv} \circ f_{2}\right)^{c v}(\mathbf{z}), f_{1}^{U}\left(\operatorname{Inv} \circ f_{2}\right)^{c c}(\mathbf{z})\right\}-\frac{f_{1}^{U}}{f_{2}^{L}}
\end{array}\right\} .
\end{aligned}
$$

Since both $\mathrm{Inv}^{\mathrm{cv}}, \mathrm{Inv}^{\mathrm{cc}}$ are decreasing in $(-\infty, 0)$ and $(0, \infty)$, by Corollary 3 we have

$$
\left(\operatorname{Inv} \circ f_{2}\right)^{c v}(\mathbf{z})=\operatorname{Inv}^{c v}\left(f_{2}^{c c}(\mathbf{z})\right), \quad\left(\operatorname{Inv} \circ f_{2}\right)^{c c}(\mathbf{z})=\operatorname{Inv}^{c c}\left(f_{2}^{c v}(\mathbf{z})\right),
$$

and thus

$$
\begin{aligned}
& \bar{g}^{c v, M C++}(\mathbf{z}) \\
& =\max \left\{\begin{array}{l}
\min \left\{\begin{array}{l}
\left.\frac{1}{f_{2}^{U}} f_{1}^{c v}(\mathbf{z}), \frac{1}{f_{2}^{U}} f_{1}^{c c}(\mathbf{z})\right\} \\
\min \left\{\frac{1}{f_{2}^{L}} f_{1}^{c v}(\mathbf{z}), \frac{1}{f_{2}^{L}} f_{1}^{c c}(\mathbf{z})\right.
\end{array}\right\}+\min \left\{f_{1}^{L} \operatorname{Inv}^{c v}\left(f_{2}^{c c}(\mathbf{z})\right), f_{1}^{L} \operatorname{Inv}^{c c}\left(f_{2}^{c v}(\mathbf{z})\right)\right\}-\frac{f_{1}^{L}}{f_{1}^{U}} \\
+\min \left\{f_{1}^{U} \operatorname{Inv}^{c v}\left(f_{2}^{c c}(\mathbf{z})\right), f_{1}^{U} \operatorname{Inv}^{c c}\left(f_{2}^{c v}(\mathbf{z})\right)\right\}-\frac{f_{1}^{U}}{f_{1}^{L}}
\end{array}\right\} .
\end{aligned}
$$

The multivariate composition theorem provides a direct method to calculate convex relaxations:

Corollary 6 Consider $Z \in \mathbb{R}^{n}$ and $G, f_{1}, f_{2}: Z \rightarrow \mathbb{R}$ such that $G(\mathbf{z})=\frac{f_{1}(\mathbf{z})}{f_{2}(\mathbf{z})}$. Suppose that interval enclosures are given for $f_{1}$ and $f_{2}$ on $Z$, i.e., bounds $f_{1}^{L}, f_{1}^{U}, f_{2}^{L}, f_{2}^{U}$ such that

$$
f_{1}^{L} \leq f_{1}(\mathbf{z}) \leq f_{1}^{U} \quad f_{2}^{L} \leq f_{2}(\mathbf{z}) \leq f_{2}^{U}
$$

and convex/concave relaxations such that

$$
f_{1}^{c v}(\mathbf{z}) \leq f_{1}(\mathbf{z}) \leq f_{1}^{c c}(\mathbf{z}) \quad f_{2}^{c v}(\mathbf{z}) \leq f_{2}(\mathbf{z}) \leq f_{2}^{c c}(\mathbf{z}) .
$$

A valid convex relaxation for $G$ on $Z$ is given by $g^{c v}$

$$
\begin{array}{r}
g^{c v}(\mathbf{z})=\min _{\substack{x_{1} \in X_{1} \\
x_{2} \in X_{2}}} \operatorname{div}_{X_{1} \times X_{2}}^{c v}\left(x_{1}, x_{2}\right) \\
\text { s.t. } \quad f_{1}^{c v}(\mathbf{z}) \leq x_{1} \leq f_{1}^{c c}(\mathbf{z}) \\
\\
f_{2}^{c v}(\mathbf{z}) \leq x_{2} \leq f_{2}^{c c}(\mathbf{z}),
\end{array}
$$

where $\operatorname{div}_{X_{1} \times X_{2}}^{c v}\left(x_{1}, x_{2}\right)$ is any valid convex relaxation of $\operatorname{div}(\cdot, \cdot)$ on $X_{1} \times X_{2}$.

Similarly, a concave relaxation is obtained by

$$
\begin{array}{r}
g^{c c}(\mathbf{z})=\max _{\substack{x_{1} \in X_{1} \\
x_{2} \in X_{2} \\
\text { s.t. }}} \operatorname{div}_{X_{1} \times X_{2}}^{c c}\left(x_{1}, x_{2}\right) \leq x_{1} \leq f_{1}^{c c}(\mathbf{z}) \\
\\
f_{2}^{c v}(\mathbf{z}) \leq x_{2} \leq f_{2}^{c c}(\mathbf{z}),
\end{array}
$$

Proposition 7 Consider the relaxation $g^{c v}$ constructed in Corollary 6 for $G(\mathbf{z})=$ $f_{1}(\mathbf{z}) / f_{2}(\mathbf{z})$ and suppose that the relaxations for $\operatorname{div}(\cdot, \cdot)$ on $X_{1} \times X_{2}$ are at least as tight as $\operatorname{div}^{c v, m c}$ and $\operatorname{div}^{c c, m c}$. Then $g^{c v}$ is at least as tight as $\bar{g}^{c v, M C++}$ as defined in Eq. (33).

Proof The proof is given in the Appendix. 


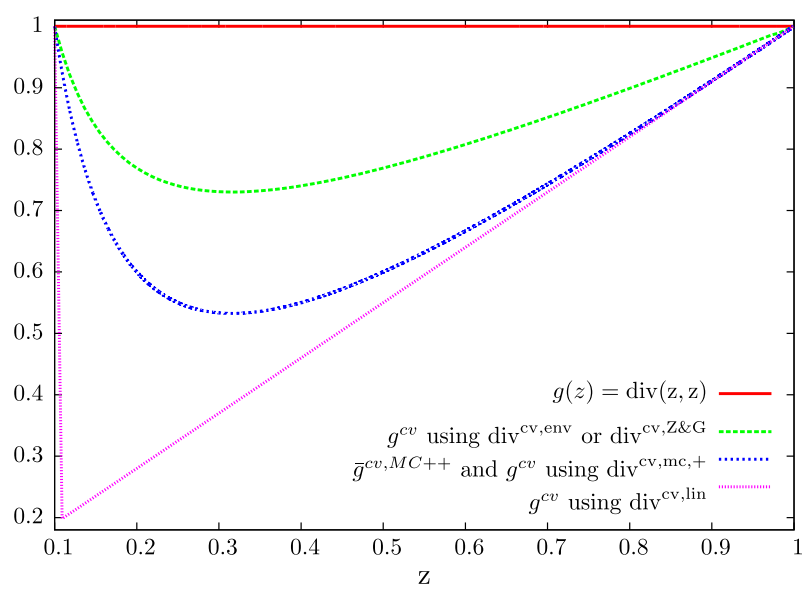

Fig. 3 Consider the trivial function $z / z=1$ on $[0.1,1]$. The inequality in Proposition 7 can be strict. The multivariate McCormick relaxations (by Theorem 6) are the same when the outer function is relaxed via $\operatorname{div}^{\mathrm{cv}, Z \& G}$ (by Zamora and Grossmann [53]), or via div ${ }^{\mathrm{cv}, \text { env }}$ (by Tawarmalani and Sahinidis [47]); the convex relaxation obtained is the tightest among the relaxations considered. The univariate McCormick relaxation

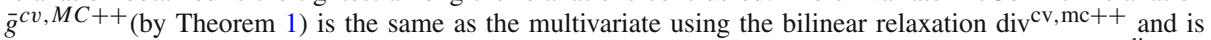
weaker than the previous ones. The loosest relaxation is obtained when the linear relaxation $\operatorname{div}^{\mathrm{cv}, \text { lin }}$ (by Tawarmalani and Sahinidis [47]) is used for the outer function in the multivariate McCormick relaxations

Figure 3 shows that the proposed relaxations can be substantially tighter than the ones obtained via the McCormick relaxations. Moreover, it shows that if weak relaxations are used for the outer function in the multivariate composition theorem, the relaxations can be weaker than the univariate McCormick relaxations.

Implementing Theorem 2 for the division of two functions is straightforward if the outer function is relaxed via (27), (28) or (29), as these relaxations are given in closed form. Subgradient computation is also straightforward and Theorem 4 can be utilized to further propagate them to outer functions.

The use of the convex envelope defined in (32) is more involved but we can use it by solving

$$
\begin{array}{ccc}
\min _{\substack{x_{1} \in X_{1} \\
x_{2} \in X_{2}}} \operatorname{div}^{c v, e n v}\left(x_{1}, x_{2}\right)=\min _{\substack{y_{p}, z_{p}, z_{c}^{e}, \lambda \\
x_{1}, x_{2}}} & z_{c}^{e} \\
\text { s.t. } f_{1}^{c v}(\mathbf{z}) \leq x_{1} \leq f_{1}^{c c}(\mathbf{z}) & \text { s.t. } & z_{p} y_{p} \geq x_{1}^{L}(1-\lambda)^{2} \\
f_{2}^{c v}(\mathbf{z}) \leq x_{2} \leq f_{2}^{c c}(\mathbf{z}) & \left(z_{c}^{e}-z_{p}\right)\left(x_{2}-y_{p}\right)=x_{1}^{U} \lambda^{2} \\
& y_{p} \geq x_{2}^{L}(1-\lambda) \\
& y_{p} \geq x_{2}-x_{2}^{U} \lambda \\
& y_{p} \leq x_{2}^{U}(1-\lambda) \\
& y_{p} \leq x_{2}-x_{2}^{L} \lambda \\
& x_{1}=x_{1}^{L}+\left(x_{1}^{U}-x_{1}^{L}\right) \lambda \\
& z_{c}^{e} \geq z_{p} \\
& \lambda \in[0,1], z_{p} \geq 0 \\
& f_{1}^{c v}(\mathbf{z}) \leq x_{1} \leq f_{1}^{c c}(\mathbf{z}) \\
& f_{2}^{c v}(\mathbf{z}) \leq x_{2} \leq f_{2}^{c c}(\mathbf{z})
\end{array}
$$


which for a given $\mathbf{z}$ can be written a semi-definite program. Similarly to the discussion of multilinear products we can obtain subgradients by using the Lagrange multipliers associated to the constraints $f_{1}^{c v}(\mathbf{z}) \leq x_{1} \leq f_{1}^{c c}(\mathbf{z}), f_{2}^{c v}(\mathbf{z}) \leq x_{2} \leq f_{2}^{c c}(\mathbf{z})$.

If the envelope is not used, one can easily take the maximum of $\operatorname{div}^{\mathrm{cv}, \mathrm{Z} \& \mathrm{~K}}$ and $\operatorname{div}^{\mathrm{cv}, \mathrm{mc}+}$. Tawarmalani and Sahinidis [47] show it can be beneficial compared to any of the two terms.

\section{Concluding remarks}

We presented a multivariate generalization of McCormick's composition theorem [23]. McCormick's results for the relaxation of composite functions with univariate outer function are the basis of so-called McCormick-relaxations, which are one of the key ideas in constructing convex relaxations in deterministic global optimization. Our generalization to multivariate outer functions results in tighter relaxations for important classes of functions including binary product of functions, the division of functions and the minimum/maximum of functions. Similarly to McCormick's composition and product theorem, the multivariate composition can be applied recursively and in fact the implementation of our result is very similar to McCormick's relaxations; many of our improvements have been implemented in both $\mathrm{MC}++[10]$ and modMC [13]. In contrast to the univariate McCormick's relaxations, our result also enables the direct relaxation of classes of functions such as multilinear products of functions. This is particularly important since in recent years many relaxations have been proposed for relatively complicated expressions and it has been shown that using this is advantageous compared to recursive application of simple rules. For instance, an important class of functions are the so-called edge-concave functions treated in $[26,44,45]$; the work presented herein can be used to obtain tight relaxations for functions that are a composition of an edge-concave outer function and an arbitrary inner function; the relaxation can be achieved via a similar reasoning to our theorems for relaxations of bilinear, multilinear and fractional terms. It would be very useful to collect all these rules and implement them in the proposed multivariate McCormick relaxations and then perform a thorough computational comparison of the advances obtained. Moreover, it would be interesting to consider other important functions found in applications, such as $\left|f_{1}(\mathbf{z})-f_{2}(\mathbf{z})\right|$ and $\left(f_{1}(\mathbf{z})-f_{2}(\mathbf{z})\right)^{2}$ which are found for instance in parameter estimation. Also, it would be interesting to consider discontinuous functions as done in [52].

Similarly to univariate McCormick relaxations, our result is also applicable to functions calculated by algorithms [30]. It is well-known that univariate McCormick relaxations are nonsmooth and recently subgradient propagation has been proposed [30]. For the proposed multivariate framework it is also possible to propagate subgradients and in fact, we provide the framework to obtain, at least in principle, the entire subdifferential.

An alternative to McCormick relaxations is the AVM. Our reformulation and generalization of McCormick's composition theorem makes the connection with this method more explicit. In particular, it illustrates that the McCormick relaxation framework can be interpreted as a decomposition method for AVM. It would be of interest to indeed utilize such decomposition methods in the AVM. Moreover, we discussed the tightness of relaxations of the AVM compared to the multivariate McCormick relaxations. In cases that common subexpressions are recognized in the AVM this can result in tighter relaxations than the McCormick relaxations [48]; the same holds for the simple recursive application of the proposed multivariate McCormick relaxations. In some cases, it is possible to introduce just enough auxiliary variables to close this gap, and it would be interesting to explore this opportunity computationally. Moreover, the proposed multivariate relaxations can result in tighter 
relaxations in specific cases by enabling the use of complicated but tight relaxations of some functions. It would be interesting to computationally compare the two methods.

Acknowledgments We thank the anonymous reviewers for the thorough review and helpful comments, in particular the reviewer that suggested the equivalence of the subgradient inequality with the linearization of the generalized benders cut in the auxiliary variable method.

Open Access This article is distributed under the terms of the Creative Commons Attribution License which permits any use, distribution, and reproduction in any medium, provided the original author(s) and the source are credited.

\section{Appendix}

Herein, proofs for three results are given.

Proof of Lemma 3

For the proof we make use of the following Lemma:

Lemma 4 ([18] Theorem 1.23) Let $T$ be a convex set and $S \subset T$ a simplex. Iff is concave on $T$ and $f^{c v, S}, f^{c v, T}$ are the convex envelopes of $f$ over $S, T$ respectively then $f^{c v, S} \geq f^{c v, T}$ on $S$.

\section{Now we prove Lemma 3}

Proof First we note that $\min ^{\mathrm{cv}}\left(\mathrm{x}_{1}, \mathrm{x}_{2}\right)$ is convex in $Z$ as the maximum of two affine functions. We have

$$
\begin{aligned}
& \min ^{\mathrm{cv}, 1}\left(\mathrm{x}_{1}^{\mathrm{L}}, \mathrm{x}_{2}^{\mathrm{L}}\right)=\min \left(x_{1}^{L}, x_{2}^{L}\right), \\
& \min ^{\mathrm{cv}, 1}\left(\mathrm{x}_{1}^{\mathrm{L}}, \mathrm{x}_{2}^{\mathrm{U}}\right)=\min \left(x_{1}^{L}, x_{2}^{U}\right), \\
& \min ^{\mathrm{cv}, 1}\left(\mathrm{x}_{1}^{\mathrm{U}}, \mathrm{x}_{2}^{\mathrm{L}}\right)=\min \left(x_{1}^{U}, x_{2}^{L}\right), \\
& \min ^{\mathrm{cv}, 1}\left(\mathrm{x}_{1}^{\mathrm{U}}, \mathrm{x}_{2}^{\mathrm{U}}\right)=\min \left(x_{1}^{U}, x_{2}^{L}\right)+\min \left(x_{1}^{L}, x_{2}^{U}\right)-\min \left(x_{1}^{L}, x_{2}^{L}\right)
\end{aligned}
$$

and

$$
\begin{aligned}
& \min ^{\mathrm{cv}, 2}\left(\mathrm{x}_{1}^{\mathrm{L}}, \mathrm{x}_{2}^{\mathrm{L}}\right)=\min \left(x_{1}^{L}, x_{2}^{U}\right)+\min \left(x_{1}^{U}, x_{2}^{L}\right)-\min \left(x_{1}^{U}, x_{2}^{U}\right), \\
& \min ^{\mathrm{cv}, 2}\left(\mathrm{x}_{1}^{\mathrm{L}}, \mathrm{x}_{2}^{\mathrm{U}}\right)=\min \left(x_{1}^{L}, x_{2}^{U}\right), \\
& \min ^{\mathrm{cv}, 2}\left(\mathrm{x}_{1}^{\mathrm{U}}, \mathrm{x}_{2}^{\mathrm{L}}\right)=\min \left(x_{1}^{L}, x_{2}^{L}\right), \\
& \min ^{\mathrm{cv}, 2}\left(\mathrm{x}_{1}^{\mathrm{U}}, \mathrm{x}_{2}^{\mathrm{U}}\right)=\min \left(x_{1}^{U}, x_{2}^{U}\right) .
\end{aligned}
$$

Without loss of generality we can assume that either $x_{1}^{L} \leq x_{1}^{U} \leq x_{2}^{L} \leq x_{2}^{U}$, if the bounds do not overlap or $x_{1}^{L} \leq x_{2}^{U}, x_{2}^{L} \leq x_{1}^{U}$, if the bounds overlap. In the former case we have

$$
\begin{aligned}
& \min ^{\mathrm{cv}, 1}\left(\mathrm{x}_{1}^{\mathrm{U}}, \mathrm{x}_{2}^{\mathrm{U}}\right)=\mathrm{x}_{1}^{\mathrm{U}}+\mathrm{x}_{1}^{\mathrm{L}}-\mathrm{x}_{1}^{\mathrm{L}}=\mathrm{x}_{1}^{\mathrm{U}}=\min \left(\mathrm{x}_{1}^{\mathrm{U}}, \mathrm{x}_{2}^{\mathrm{U}}\right), \\
& \min ^{\mathrm{cv}, 2}\left(\mathrm{x}_{1}^{\mathrm{L}}, \mathrm{x}_{2}^{\mathrm{L}}\right)=\mathrm{x}_{1}^{\mathrm{L}}+\mathrm{x}_{1}^{\mathrm{U}}-\mathrm{x}_{1}^{\mathrm{U}}=\mathrm{x}_{1}^{\mathrm{L}}=\min \left(\mathrm{x}_{1}^{\mathrm{L}}, \mathrm{x}_{2}^{\mathrm{L}}\right) .
\end{aligned}
$$


In the latter case

$$
\begin{aligned}
& \min ^{\mathrm{cv}, 1}\left(\mathrm{x}_{1}^{\mathrm{U}}, \mathrm{x}_{2}^{\mathrm{U}}\right)=\mathrm{x}_{2}^{\mathrm{L}}+\mathrm{x}_{1}^{\mathrm{L}}-\min \left(\mathrm{x}_{1}^{\mathrm{L}}, \mathrm{x}_{2}^{\mathrm{L}}\right)=\max \left(\mathrm{x}_{1}^{\mathrm{L}}, \mathrm{x}_{2}^{\mathrm{L}}\right) \leq \min \left(\mathrm{x}_{1}^{\mathrm{U}}, \mathrm{x}_{2}^{\mathrm{U}}\right), \\
& \min ^{\mathrm{cv}, 2}\left(\mathrm{x}_{1}^{\mathrm{L}}, \mathrm{x}_{2}^{\mathrm{L}}\right)=\mathrm{x}_{2}^{\mathrm{L}}+\mathrm{x}_{1}^{\mathrm{L}}-\min \left(\mathrm{x}_{1}^{\mathrm{U}}, \mathrm{x}_{2}^{\mathrm{U}}\right) \leq \mathrm{x}_{2}^{\mathrm{L}}+\mathrm{x}_{1}^{\mathrm{L}}-\max \left(\mathrm{x}_{1}^{\mathrm{L}}, \mathrm{x}_{2}^{\mathrm{L}}\right) \leq \min \left(\mathrm{x}_{1}^{\mathrm{L}}, \mathrm{x}_{2}^{\mathrm{L}}\right) .
\end{aligned}
$$

Since $\min \left(x_{1}, x_{2}\right)$ is concave, $\min ^{\mathrm{cv}, 1}, \min ^{\mathrm{cv}, 2}$ affine and $\min ^{\mathrm{cv}, 1}\left(\mathrm{x}_{1}, \mathrm{x}_{2}\right) \leq \min \left(\mathrm{x}_{1}, \mathrm{x}_{2}\right)$, $\min ^{\mathrm{cv}, 2}\left(\mathrm{x}_{1}, \mathrm{x}_{2}\right) \leq \min \left(\mathrm{x}_{1}, \mathrm{x}_{2}\right)$ for all vertices of the box $Z$, it follows that $\min ^{\mathrm{cv}, 1}, \min ^{\mathrm{cv}, 2}$, and thus also $\min ^{\mathrm{cv}}$ are convex underestimators of $\min \left(x_{1}, x_{2}\right)$ on $Z$.

Also, if $S_{1}$ is the simplex defined by the points $\left\{\left(x_{1}^{L}, x_{2}^{L}\right),\left(x_{1}^{L}, x_{2}^{U}\right),\left(x_{1}^{U}, x_{2}^{L}\right)\right\}$ and since $\min ^{\mathrm{cv}, 1}\left(\mathrm{x}_{1}, \mathrm{x}_{2}\right)=\min \left(\mathrm{x}_{1}, \mathrm{x}_{2}\right)$ for all vertices of $S_{1}$, it follows, see for example [18], that $\min ^{\mathrm{cv}, 1}\left(\mathrm{x}_{1}, \mathrm{x}_{2}\right)$ is the convex envelope of $\min \left(x_{1}, x_{2}\right)$ in $S_{1}$. Similarly if $S_{2}$ is the simplex defined by the points $\left\{\left(x_{1}^{L}, x_{2}^{U}\right),\left(x_{1}^{U}, x_{2}^{L}\right),\left(x_{1}^{U}, x_{2}^{U}\right)\right\}$ and since $\min ^{\mathrm{cv}, 2}\left(\mathrm{x}_{1}, \mathrm{x}_{2}\right)=$ $\min \left(\mathrm{x}_{1}, \mathrm{x}_{2}\right)$ for all vertices of $S_{2}$, it follows that $\min ^{\mathrm{cv}, 2}\left(\mathrm{x}_{1}, \mathrm{x}_{2}\right)$ is the convex envelope of $\min \left(x_{1}, x_{2}\right)$ in $S_{2}$.

From Lemma 4, if $F_{Z}^{c v}$ is the convex envelope of $\min \left(x_{1}, x_{2}\right)$ on $Z$, then we have

$$
\begin{aligned}
& F_{Z}^{c v}(\mathbf{z}) \leq \min ^{\mathrm{cv}, 1}(\mathbf{z}) \text { for all } \quad \mathbf{z} \in \mathrm{S}_{1}, \\
& F_{Z}^{c v}(\mathbf{z}) \leq \min ^{\mathrm{cv}, 2}(\mathbf{z}) \text { for all } \quad \mathbf{z} \in \mathrm{S}_{2} .
\end{aligned}
$$

Thus

$$
F_{Z}^{c v}(\mathbf{z}) \leq \max \left(\min ^{\mathrm{cv}, 1}(\mathbf{z}), \min ^{\mathrm{cv}, 2}(\mathbf{z})\right) \text { for all } \mathbf{z} \in S_{1} \cup S_{2}=Z
$$

and $\min ^{\mathrm{cv}}$ is the convex envelope of $\min \left(x_{1}, x_{2}\right)$ on $Z$.

The proof for the concave envelope of $\max \left(x_{1}, x_{2}\right)$ is similar and is omitted.

\section{Proof of Proposition 6}

Proof Since the negative absolute value is concave and piecewise affine linear, its envelope is the secant. Thus, application of McCormick's composition Theorem 1 as reformulated in (2) gives

$$
\begin{gathered}
\bar{g}_{1}^{c v, a b s}(\mathbf{z})=\min _{w} \min ^{\mathrm{cv}, \mathrm{abs}}(\mathbf{z}, \mathrm{w}) \\
\text { s.t. } f_{1}^{c v}(\mathbf{z})-f_{2}^{c c}(\mathbf{z}) \leq w \leq f_{1}^{c c}(\mathbf{z})-f_{2}^{c v}(\mathbf{z}) \\
\quad w^{L} \leq w \leq w^{U},
\end{gathered}
$$

where $w^{L}=f_{1}^{L}-f_{2}^{U}$ and $w^{U}=f_{1}^{U}-f_{2}^{L}$, with

$$
\min ^{\mathrm{cv}, \mathrm{abs}}(\mathbf{z}, \mathrm{w})=0.5\left(\mathrm{f}_{1}^{\mathrm{cv}}(\mathbf{z})+\mathrm{f}_{2}^{\mathrm{cv}}(\mathbf{z})+\left|\mathrm{w}^{\mathrm{L}}\right|+\frac{-\left|\mathrm{w}^{\mathrm{U}}\right|+\left|\mathrm{w}^{\mathrm{L}}\right|}{\mathrm{w}^{\mathrm{U}}-\mathrm{w}^{\mathrm{L}}}\left(\mathrm{w}-\mathrm{w}^{\mathrm{L}}\right)\right) .
$$

On the other hand, Theorem 2 gives a convex relaxation for $g_{1}$ on $Z$

$$
\begin{aligned}
& g_{1}^{c v}(\mathbf{z})=\min _{\mathbf{x}} \min ^{c v}(\mathbf{x}) \\
& \text { s.t. } f_{i}^{c v}(\mathbf{z}) \leq x_{i} \leq f_{i}^{c c}(\mathbf{z}) \\
& \quad x_{i}^{L} \leq x_{i} \leq x_{i}^{U}, \quad i=1,2,
\end{aligned}
$$

where $x_{i}^{L}=f_{i}^{L}, x_{i}^{U}=f_{i}^{U}$ and $\min ^{\mathrm{cv}}$ is the convex envelope of $\min (\cdot, \cdot)$ on $X=\left(x_{1}^{L}, x_{1}^{U}\right) \times$ $\left(x_{2}^{L}, x_{2}^{U}\right)$. We will show that (36) has an optimal value smaller or equal to the optimal value of (37) for an arbitrary but fixed $\mathbf{z}$, and thus $\min ^{\mathrm{cv}, \mathrm{abs}}(\mathbf{z}) \leq \min ^{\mathrm{cv}}(\mathbf{z})$. To do so, first we 
reformulate (36) by introducing two new variables $x_{1}, x_{2}$ with $w=x_{1}-x_{2}$ and eliminate $w$ obtaining

$$
\begin{aligned}
\bar{g}_{1}^{c v, a b s}(\mathbf{z})= & \min _{x_{1}, x_{2}} \min ^{\mathrm{cv}, \mathrm{abs}}\left(\mathbf{z}, \mathrm{x}_{1}-\mathrm{x}_{2}\right) \\
& \text { s.t. } f_{1}^{c v}(\mathbf{z})-f_{2}^{c c}(\mathbf{z}) \leq x_{1}-x_{2} \leq f_{1}^{c c}(\mathbf{z})-f_{2}^{c c}(\mathbf{z}) \\
& \quad w^{L} \leq x_{1}-x_{2} \leq w^{U} .
\end{aligned}
$$

which is equivalent with (36). Next we show that the optimization problem at the right hand of (38) is a relaxation of the optimization problem at the right hand of (37) and thus has a smaller optimal value.

First we will show that any feasible point of (37) is also feasible in (38). Indeed, take any feasible $\left(x_{1}, x_{2}\right)$. By feasibility we have

$$
f_{1}^{L} \leq x_{1} \leq f_{1}^{U}, \quad f_{2}^{L} \leq x_{2} \leq f_{2}^{U}
$$

and thus

$$
f_{1}^{L}-f_{2}^{U} \leq x_{1}-x_{2} \leq f_{1}^{U}-f_{2}^{L}
$$

Similarly by feasibility

$$
f_{1}^{c v}(\mathbf{z}) \leq x_{1} \leq f_{1}^{c c}(\mathbf{z}), \quad f_{2}^{c v}(\mathbf{z}) \leq x_{2} \leq f_{2}^{c c}(\mathbf{z})
$$

and thus

$$
f_{1}^{c v}(\mathbf{z})-f_{2}^{c c}(\mathbf{z}) \leq x_{1}-x_{2} \leq f_{1}^{c c}(\mathbf{z})-f_{2}^{c v}(\mathbf{z})
$$

and $\left(x_{1}, x_{2}\right)$ is also feasible in (38).

It remains to show that the objective function of (38) is an underestimate of (37). Take any $\left(x_{1}, x_{2}\right)$ which is feasible in (37). By construction of $\min ^{\mathrm{cv} \text {, abs }}$ we have

$$
\min ^{\mathrm{cv}, \mathrm{abs}}\left(\mathbf{z}, \mathrm{x}_{1}-\mathrm{x}_{2}\right) \leq 0.5\left(\mathrm{f}_{1}^{\mathrm{cv}}(\mathbf{z})+\mathrm{f}_{2}^{\mathrm{cv}}(\mathbf{z})-\left|\mathrm{x}_{1}-\mathrm{x}_{2}\right|\right) .
$$

By feasibility of $\left(x_{1}, x_{2}\right)$ in (36) we also have

$$
f_{1}^{c v}(\mathbf{z}) \leq x_{1}, \quad f_{2}^{c v}(\mathbf{z}) \leq x_{2}
$$

and thus combining the last two inequalities we also obtain

$$
\min ^{\mathrm{cv}, \mathrm{abs}}\left(\mathbf{z}, \mathrm{x}_{1}-\mathrm{x}_{2}\right) \leq \mathrm{x}_{1}+\mathrm{x}_{2}-\left|\mathrm{x}_{1}-\mathrm{x}_{2}\right|=\min \left(\mathrm{x}_{1}, \mathrm{x}_{2}\right)
$$

or $\min ^{\mathrm{cv}, \text { abs }}(\mathbf{z}, \cdot)$ underestimates $\min (\cdot, \cdot)$ on $X$. Moreover, $\min ^{\mathrm{cv}, \mathrm{abs}}(\mathrm{z}, \cdot)$ is affine linear and thus also convex on $X$. Since $\min ^{\mathrm{cv}}$ is the convex envelope of $\min (\cdot, \cdot)$ on $X$ we directly obtain

$$
\min ^{\mathrm{cv}, \mathrm{abs}}\left(\mathbf{z}, \mathrm{w}=\mathrm{x}_{1}-\mathrm{x}_{2}\right) \leq \min ^{\mathrm{cv}}\left(\mathrm{x}_{1}, \mathrm{x}_{2}\right)
$$

and the result follows.

The result for the concave envelope is analogous and so are the results for the max. 


\section{Proof of Proposition 7}

Proof Assume first that $\operatorname{div}^{c v, m c}$ is used for $\operatorname{div}_{X_{1} \times X_{2}}^{c v}$. In that case the relaxations are obtained by

$$
\begin{aligned}
& g^{c v}(\mathbf{z})=\min _{\substack{x_{1} \in X_{1} \\
x_{2} \in X_{2}}} \operatorname{div}^{c v, m c}\left(x_{1}, x_{2}\right) \\
& \text { s.t. } \quad f_{1}^{c v}(\mathbf{z}) \leq x_{1} \leq f_{1}^{c c}(\mathbf{z}) \\
& f_{2}^{c v}(\mathbf{z}) \leq x_{2} \leq f_{2}^{c c}(\mathbf{z})
\end{aligned}
$$

or substituting (29) for $\operatorname{div}^{c v, m c}\left(x_{1}, x_{2}\right)$ and the implied bounds on which the relaxation is computed $f_{1}^{L} \leq x_{1} \leq f_{1}^{U}, f_{2}^{L} \leq x_{2} \leq f_{2}^{U}$ we obtain

$$
\begin{aligned}
& g^{c v}(\mathbf{z})=\min _{\substack{x_{1} \in X_{1} \\
x_{2} \in X_{2}}} \max \left\{\begin{array}{l}
\frac{1}{f_{2}^{U}} x_{1}+\min \left\{f_{1}^{L} \operatorname{Inv}^{c v}\left(x_{2}\right), f_{1}^{L} \operatorname{Inv}^{c c}\left(x_{2}\right)\right\}-\frac{f_{1}^{L}}{f_{2}^{U}}, \\
\frac{1}{f_{2}^{L}} x_{1}+\min \left\{f_{1}^{U} \operatorname{Inv}^{c v}\left(x_{2}\right), f_{1}^{U} \operatorname{Inv}^{c c}\left(x_{2}\right)\right\}-\frac{f_{1}^{U}}{f_{2}^{L}} .
\end{array}\right\} \\
& \text { s.t. } f_{1}^{c v}(\mathbf{z}) \leq x_{1} \leq f_{1}^{c c}(\mathbf{z}) \\
& f_{2}^{c v}(\mathbf{z}) \leq x_{2} \leq f_{2}^{c c}(\mathbf{z})
\end{aligned}
$$

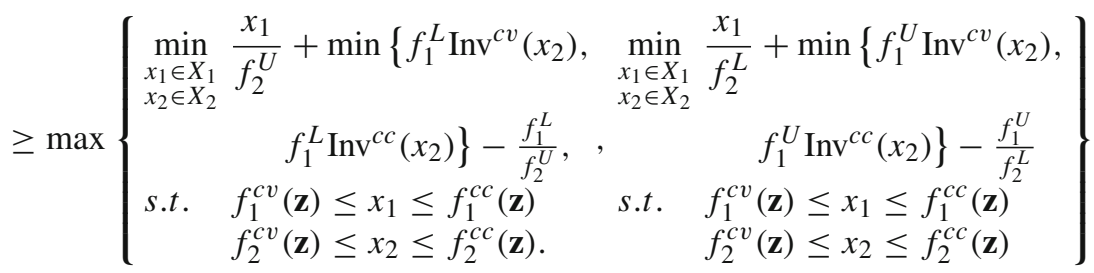

where the inequality was obtained (similarly to the proof of proposition 3) by interchanging the min and max operators.

Since the inner problems are separable in $x_{1}, x_{2}$ we have

$$
g^{c v}(\mathbf{z}) \geq \max \left\{\zeta_{1}(\mathbf{z})+\zeta_{2}(\mathbf{z})-\frac{f_{1}^{L}}{f_{2}^{U}}, \zeta_{3}(\mathbf{z})+\zeta_{4}(\mathbf{z})-\frac{f_{1}^{U}}{f_{2}^{L}}\right\}
$$

with

$$
\begin{aligned}
& \zeta_{1}(\mathbf{z})=\min _{x_{1} \in X_{1}} \quad \frac{1}{f_{2}^{U}} x_{1} \quad, \quad \zeta_{2}(\mathbf{z})=\min _{x_{2} \in X_{2}} \min \left\{f_{1}^{L} \operatorname{Inv}^{c v}\left(x_{2}\right), f_{1}^{L} \operatorname{Inv}^{c c}\left(x_{2}\right)\right\} \\
& \text { s.t. } f_{1}^{c v}(\mathbf{z}) \leq x_{1} \leq f_{1}^{c c}(\mathbf{z}) \quad \text { s.t. } \quad f_{2}^{c v}(\mathbf{z}) \leq x_{2} \leq f_{2}^{c c}(\mathbf{z}) \\
& \zeta_{3}(\mathbf{z})=\min _{x_{1} \in X_{1}} \quad \frac{1}{f_{2}^{L}} x_{1} \quad \zeta_{4}(\mathbf{z})=\min _{x_{2} \in X_{2}} \min \left\{f_{1}^{U} \operatorname{Inv}^{c v}\left(x_{2}\right), f_{1}^{U} \operatorname{Inv}^{c c}\left(x_{2}\right)\right\} \\
& \text { s.t. } f_{1}^{c v}(\mathbf{z}) \leq x_{1} \leq f_{1}^{c c}(\mathbf{z}) \quad \text { s.t. } \quad f_{2}^{c v}(\mathbf{z}) \leq x_{2} \leq f_{2}^{c c}(\mathbf{z})
\end{aligned}
$$

We have

$$
\zeta_{1}(\mathbf{z})=\min \left\{\frac{1}{f_{2}^{U}} f_{1}^{c v}(\mathbf{z}), \frac{1}{f_{2}^{U}} f_{1}^{c c}(\mathbf{z})\right\}, \quad \zeta_{3}(\mathbf{z})=\min \left\{\frac{1}{f_{2}^{L}} f_{1}^{c v}(\mathbf{z}), \frac{1}{f_{2}^{L}} f_{1}^{c c}(\mathbf{z})\right\} .
$$

Also, we have

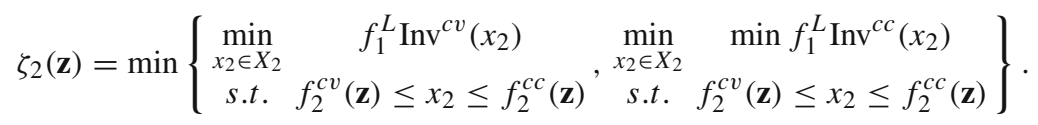


We treat separately the cases $f_{1}^{L} \geq 0$ versus $f_{1}^{L}<0$. If $f_{1}^{L} \geq 0$ since both Inv ${ }^{\mathrm{cv}}$, Inv ${ }^{\mathrm{cc}}$ are decreasing monotonically we have

$$
\zeta_{2}(\mathbf{z})=\min \left\{\begin{array}{c}
f_{1}^{L} \operatorname{Inv}^{c v}\left(f_{2}^{c c}(\mathbf{z})\right), \\
f_{1}^{L} \operatorname{Inv}^{c c}\left(f_{2}^{c c}(\mathbf{z})\right)
\end{array}\right\}=f_{1}^{L} \operatorname{Inv}^{c v}\left(f_{2}^{c c}(\mathbf{z})\right)
$$

But

$$
f_{1}^{L} \operatorname{Inv}^{c v}\left(f_{2}^{c c}(\mathbf{z})\right) \leq f_{1}^{L} \operatorname{Inv}^{c v}\left(f_{2}^{c v}(\mathbf{z})\right) \leq f_{1}^{L} \operatorname{Inv}^{c c}\left(f_{2}^{c v}(\mathbf{z})\right)
$$

and therefore

$$
\zeta_{2}(\mathbf{z})=\min \left\{\begin{array}{l}
f_{1}^{L} \operatorname{Inv}^{c v}\left(f_{2}^{c c}(\mathbf{z})\right) \\
f_{1}^{L} \operatorname{Inv}^{c c}\left(f_{2}^{c v}(\mathbf{z})\right)
\end{array}\right\} .
$$

If on the other hand $f_{1}^{L}<0$ since both $-\mathrm{Inv}^{\mathrm{cv}},-\mathrm{Inv}{ }^{\mathrm{cc}}$ are increasing monotonically we have

$$
\zeta_{2}(\mathbf{z})=\min \left\{\begin{array}{l}
f_{1}^{L} \operatorname{Inv}^{c v}\left(f_{2}^{c v}(\mathbf{z})\right), \\
f_{1}^{L} \operatorname{Inv}^{c c}\left(f_{2}^{c v}(\mathbf{z})\right)
\end{array}\right\}=f_{1}^{L} \operatorname{Inv}^{c c}\left(f_{2}^{c v}(\mathbf{z})\right) .
$$

In this case due to negativity of $f_{1}^{L}$ there holds

$$
f_{1}^{L} \operatorname{Inv}^{c c}\left(f_{2}^{c v}(\mathbf{z})\right) \leq f_{1}^{L} \operatorname{Inv}^{c c}\left(f_{2}^{c c}(\mathbf{z})\right) \leq f_{1}^{L} \operatorname{Inv}^{c v}\left(f_{2}^{c c}(\mathbf{z})\right)
$$

and we obtain again

$$
\zeta_{2}(\mathbf{z})=\min \left\{\begin{array}{l}
f_{1}^{L} \operatorname{Inv}^{c v}\left(f_{2}^{c c}(\mathbf{z})\right) \\
f_{1}^{L} \operatorname{Inv}^{c c}\left(f_{2}^{c v}(\mathbf{z})\right)
\end{array}\right\} .
$$

Therefore, we have established that Eq. (41) holds independently of the sign of $f_{1}^{L}$. By a similar reasoning we deduce that

$$
\zeta_{4}(\mathbf{z})=\min \left\{\begin{array}{l}
f_{1}^{U} \operatorname{Inv}^{c v}\left(f_{2}^{c c}(\mathbf{z})\right) \\
f_{1}^{U} \operatorname{Inv}^{c c}\left(f_{2}^{c v}(\mathbf{z})\right)
\end{array}\right\} .
$$

From inequality (39) and Eqs. (33),(40),(41),(42) we obtain

$$
g^{c v}(\mathbf{z}) \geq \bar{g}^{c v, M C++}(\mathbf{z}) .
$$

If the available relaxations of $\operatorname{div}(\cdot, \cdot)$ on $X_{1} \times X_{2}$ are tighter than $\operatorname{div}^{c v, m c}$ and $\operatorname{div}^{c c, m c}$ the resulting relaxation has to be even tighter.

\section{References}

1. Adjiman, C.S., Floudas, C.A.: Rigorous convex underestimators for general twice-differentiable problems. J. Glob. Optim. 9(1), 23-40 (1996)

2. Akrotirianakis, I.G., Floudas, C.A.: A new class of improved convex underestimators for twice continuously differentiable constrained NLPs. J. Glob. Optim. 30(4), 367-390 (2004)

3. Al-Khayyal, F.A., Falk, J.E.: Jointly constrained biconvex programming. Math. Oper. Res. 8(2), 273-286 (1983)

4. Bao, X., Khajavirad, A., Sahinidis, N.V., Tawarmalani, M.: Global optimization of nonconvex problems with multilinear intermediates. Math. Program. Comput. (2013, submitted for publication)

5. Belotti, P., Cafieri, S., Lee, J., Liberti, L., Miller, A.: On the composition of convex envelopes for quadrilinear terms. In: Chinchuluun, A., Pardalos, P.M., Enkhbat, R., Pistikopoulos, E.N. (eds.) Optimization, Simulation, and Control, vol. 76 of Springer Optimization and Its Applications, pp. 1-16. Springer, New York (2013) 
6. Birge, J.R.: Decomposition and partitioning methods for multistage stochastic linear programs. Oper. Res. 33(5), 989-1007 (1985)

7. Bompadre, A., Mitsos, A.: Convergence rate of McCormick relaxations. J. Glob. Optim. 52(1), 1-28 (2012)

8. Boyd, S.P., Vandenberghe, L.: Convex Optimization. Cambridge University Press, Cambridge, UK (2004)

9. Cafieri, S., Lee, J., Liberti, L.: On convex relaxations of quadrilinear terms. J. Glob. Optim. 47, 661-685 (2010)

10. Chachuat, B.: $\mathrm{MC}++$ : a versatile library for bounding and relaxation of factorable functions. http://www3. imperial.ac.uk/environmentenergyoptimisation/software (2013)

11. Chachuat, B., Singer, A.B., Barton, P.I.: Global mixed integer dynamic optimization. AIChE J. 51(8), 2235-2253 (2005)

12. Chachuat, B., Singer, A.B., Barton, P.I.: Global methods for dynamic optimization and mixed-integer dynamic optimization. Ind. Eng. Chem. Res. 45(25), 8373-8392 (2006)

13. Corbett, C., Maier, M., Beckers, M., Naumann, U., Ghobeity, A., Mitsos, A.: Compiler-generated subgradient code for mccormick relaxations. Technical Report AIB 2011-25, RWTH Aachen. http://www. stce.rwth-aachen.de/software/modMC.html (2011)

14. Freund, R.: Nonlinear Programming. Lecture Notes, MIT (2012)

15. Geoffrion, A.M.: Generalized benders decomposition. J. Optim. Theory Appl. 10(4), 237-260 (1972)

16. Hiriart-Urruty, J.-B., Lemaréchal, C.: Convex Analysis and Minimization Algorithms I Fundamentals. Springer, Berlin (1993)

17. Hiriart-Urruty, J.-B., Lemaréchal, C.: Fundamentals of Convex Analysis. Springer, Berlin (2001)

18. Horst, R., Pardalos, P.M., Thoai, N.V.: Introduction to Global Optimization. Springer, Netherlands (2000)

19. Khajavirad, A., Sahinidis, N.V.: Convex envelopes of products of convex and component-wise concave functions. J. Glob. Optim. 52(3), 391-409 (2012)

20. Liberti, L., Pantelides, C.C.: Convex envelopes of monomials of odd degree. J. Glob. Optim. 25(2), 157-168 (2003)

21. Maranas, C.D., Floudas, C.A.: A global optimization approach for Lennard-Jones microclusters. J. Chem. Phys. 97(10), 7667-7678 (1992)

22. Maranas, C.D., Floudas, C.A.: Finding all solutions of nonlinearly constrained systems of equations. J. Glob. Optim. 7(2), 143-182 (1995)

23. McCormick, G.P.: Computability of global solutions to factorable nonconvex programs: part I convex underestimating problems. Math. Program. 10(1), 147-175 (1976)

24. McCormick, G.P.: Nonlinear Programming: Theory, Algorithms, and Applications. Wiley, New York (1983)

25. Meyer, C.A., Floudas, C.A.: Trilinear monomials with mixed sign domains: facets of the convex and concave envelopes. J. Glob. Optim. 29(2), 125-155 (2004)

26. Meyer, C.A., Floudas, C.A.: Convex envelopes for edge-concave functions. Math. Program. 103(2), 207224 (2005)

27. Misener, R., Floudas, C.A.: A framework for globally optimizing mixed-integer signomial programs. J. Optim. Theory Appl. (2013, in press) doi:10.1007/s10957-013-0396-3

28. Misener, R., Floudas, C.A.: GloMIQO: global mixed-integer quadratic optimizer. J. Glob. Optim. 57(1), 3-50 (2013)

29. Misener, R., Floudas, C.A.: ANTIGONE: algorithms for continuous/integer global optimization of nonlinear equations. J. Glob. Optim. (2014, accepted for publication)

30. Mitsos, A., Chachuat, B., Barton, P.I.: McCormick-based relaxations of algorithms. SIAM J. Optim. 20(2), 573-601 (2009)

31. Nemirovski, A.: Efficient Methods in Convex Programming. http://www2.isye.gatech.edu/nemirovs/ Lect_EMCO.pdf (2005)

32. O'Neill, R.P.: Nested decomposition of multistage convex programs. SIAM J. Control Optim. 14(3), 409-418 (1976)

33. Quesada, I., Grossmann, I.E.: A global optimization algorithm for linear fractional and bilinear programs. J. Glob. Optim. 6(1), 39-76 (1995)

34. Rikun, A.D.: A convex envelope formula for multilinear functions. J. Glob. Optim. 10(4), 425-437 (1997)

35. Ryoo, H.S., Sahinidis, N.V.: A branch-and-reduce approach to global optimization. J. Glob. Optim. 8(2), 107-138 (1996)

36. Sahinidis, N.V.: BARON: a general purpose global optimization software package. J. Glob. Optim. 8(2), 201-205 (1996)

37. Sahlodin, A.M., Chachuat, B.: Convex/concave relaxations of parametric ODEs using taylor models. Comput. Chem. Eng. 35(5), 844-857 (2011) 
38. Scott, J.K., Stuber, M.D., Barton, P.I.: Generalized mccormick relaxations. J. Glob. Optim. 51(4), 569-606 (2011)

39. Singer, A.B., Barton, P.I.: Global solution of optimization problems with parameter-embedded linear dynamic systems. J. Optim. Theory Appl. 121(3), 613-646 (2004)

40. Singer, A.B., Barton, P.I.: Bounding the solutions of parameter dependent nonlinear ordinary differential equations. SIAM J. Sci. Comput. 27(6), 2167-2182 (2006)

41. Smith, E., Pantelides, C.C.: A symbolic reformulation/spatial branch-and-bound algorithm for the global optimisation of nonconvex minlps. Comput. Chem. Eng. 23(4-5), 457-478 (1999)

42. Smith, E., Pantelides, C.C.: Global optimisation of nonconvex minlps. Comput. Chem. Eng. 21, S791S796 (1997)

43. Stuber, M.D., Barton, P.I.: Robust simulation and design using semi-infinite programs with implicit functions. Int. J. Reliab. Saf. 5, 378-397 (2011)

44. Tardella, F.: On the existence of polyhedral convex envelopes. In: Floudas, C.A., Pardalos, P. (eds.) Frontiers in Global Optimization, vol. 74 of Nonconvex Optimization and Its Applications, pp. 563-573 (2003)

45. Tardella, F.: Existence and sum decomposition of vertex polyhedral convex envelopes. Optim. Lett. 2(3), 363-375 (2008)

46. Tawarmalani, M., Sahinidis, N.V.: Semidefinite relaxations of fractional programs via novel convexification techniques. J. Glob. Optim. 20(2), 133-154 (2001)

47. Tawarmalani, M., Sahinidis, N.V.: Convex extensions and envelopes of lower semi-continuous functions. Math. Program. 93(2), 247-263 (2002)

48. Tawarmalani, M., Sahinidis, N.V.: Convexification and Global Optimization in Continuous and MixedInteger Nonlinear Programming: Theory, Algorithms, Software, and Applications, . Kluwer Academic Publishers, Dortrecht, The Netherlands (2002)

49. Tawarmalani, M., Sahinidis, N.V.: A polyhedral branch-and-cut approach to global optimization. Math. Program. 103(2), 225-249 (2005)

50. Tawarmalani, M., Sahinidis, N.V.: Global optimization of mixed-integer nonlinear programs: a theoretical and computational study. Math. Program. 99(3), 563-591 (2004)

51. Von Neumann, J., Morgenstern, O.: Theory of Games and Economic Behavior. Princeton University Press, Princeton (1953)

52. Wechsung, A., Barton, P.I.: Global Optimization of Discontinuous Functions. In: AIChE annual meeting (2010)

53. Zamora, J.M., Grossmann, I.E.: A global MINLP optimization algorithm for the synthesis of heat exchanger networks with no stream splits. Comput. Chem. Eng. 22(3), 367-384 (1998)

54. Zamora, J.M., Grossmann, I.E.: A branch and contract algorithm for problems with concave univariate, bilinear and linear fractional terms. J. Glob. Optim. 14(3), 217-249 (1999) 